\title{
Ubber die Darstellung der Zahlen durch einige quaternäre quadratische Formen
}

von

\author{
G. LoMadse (Tbilissi)
}

§ 1. In der vorliegenden Arbeit bezeichnen die Buchstaben $M, N$, $a, d, q, r$ natürliche Zahlen (in $\S 6$ bezeichnet jedoch $q$ beliebige ganze Zahlen); $b, u, v$ ungerade natürliche Zahlen; $p$ Primzahlen; $x, \lambda$ nichtnegative ganze Zahlen; $H, c, g, h, j, k, l, m, n, x, y, \alpha, \beta, \gamma, \delta$ ganze Zahlen; $A, \mu, \nu, \varrho, \xi, \eta, \omega, t, w, W$ reelle Zahlen; $z, \zeta, \tau, A, B, C, D$ komplexe Zahlen, wobei $\operatorname{Im} \tau>0$.

Mit $K$ werden positive Zahlen bezeichnet, die an den betreffenden Stellen definiert sind.

Diese Buchstaben werden nötigenfalls mit Indizes oder Strichen versehen.

$(h, m)$ ist der größte gemeinsame Teiler von $h$ und $m$.

$d \mid m$ bedeutet, daß $d$ in $m$ als Teiler aufgeht; $d \times m$, daß $d$ nicht in $m$ aufgeht; $p^{\lambda} \| m$, daß $p^{\lambda} \mid m$, aber $p^{\lambda+1} \times m$.

$\left(\frac{h}{u}\right)$ ist das Jacobische Symbol für $(h, u)=1, u>1$; ist Null für $(h, u)>1$; ist Eins für $u=1$.

Weiter sei

$$
\begin{gathered}
e(z)=\exp 2 \pi i z, \quad Q=e\left(\frac{\tau}{4 a a^{\prime}}\right), \\
\operatorname{sgn} \omega=\left\{\begin{array}{lll}
0 & \text { für } & \omega=0, \\
\frac{\omega}{|\omega|} & \text { für } & \omega \neq 0 .
\end{array}\right.
\end{gathered}
$$

In der Summe $\sum$ durchläuft $h$ ein vollständiges Restsystem $\bmod q$, in der Summe $\sum_{h \bmod q}^{h \bmod q}$ ein reduziertes System. Leere Summen sind gleich Null zu setzen, leere Produkte gleich Eins. 
$f(\xi) \sim \varphi(\xi)$ bedeutet, da $\beta \frac{f(\xi)}{\varphi(\xi)} \rightarrow 1$, wenn $\xi$ gegen die gegebene

Grenze strebt.

$\mu(q)$ ist die Möbiussche Funktion.

Ferner sei

$$
\sigma(u)=\sum_{d \mid u} d
$$

$$
S(h, q)=\sum_{j \bmod q} e\left(\frac{h j^{2}}{q}\right) \quad \text { (die Gaußsche Summe) }
$$

$$
c(h, q)=\sum_{j \bmod q}^{\prime} e\left(\frac{h j}{q}\right) \quad \text { (die Ramanujansche Summe) }
$$

(1.4) $\vartheta_{g h}(z \mid \tau ; c, N)=\sum_{\substack{m=-\infty \\ m \equiv c(\bmod N)}}^{\infty} e\left(\frac{h(m-c)}{2 N}\right) e\left(\frac{\left(m+\frac{1}{2} g\right)^{2}}{2 N} \tau\right) e\left(\left(m+\frac{1}{2} g\right) z\right)$

(die Thetafunktion mit Charakteristiken $g, h$ ),

$$
\vartheta_{g h}(\tau ; c, N)=\vartheta_{g h}(0 \mid \tau ; c, N)
$$

§ 2. $r(n)$ bezeichne die Anzahl der Darstellungen von $n>0$ durch die Form $a\left(x_{1}^{2}+x_{2}^{2}\right)+a^{\prime}\left(x_{3}^{2}+x_{4}^{2}\right)$, d. h. die Lösungszahl der Gleichung

$$
n=a\left(x_{1}^{2}+x_{2}^{2}\right)+a^{\prime}\left(x_{3}^{2}+x_{4}^{2}\right),
$$

bei gegebenen $a, a^{\prime}$.

Kloosterman [2] erhielt mittels Modulfunktionen und Jacobischer Thetafunktionen exakte Formeln für $r(n)$, wenn $a=1 ; a^{\prime}=5,6,7$. Im Fall $a=1, a^{\prime}=7$ führt Kloosterman die Rechnungen eingehend durch; in den übrigen Fällen werden nur die Endformeln aufgeschrieben, unter Hinweis darauf, daß die Beweise sehr kompliziert sind. Die entsprechende Formel für $a=1, a^{\prime}=5, n$ gerade war schon von Liouville [5] rein arithmetisch erhalten worden. Die auf Hardy und Mordell zurückgehende Kloostermansche Methode ist auf allgemeinere Formen anwendbar. Sie erfordert aber in jedem Spezialfalle komplizierte Rechnungen, die mit dem Verhalten der entsprechenden Modulfunktion in ihrem Fundamentalbereich verknüpft sind.

Streefkerk [8] erhielt durch Anwendung gewisser ganzen Modulformen exakte Formeln für die Anzahl der Darstellungen durch Summen einiger verallgemeinerten Polygonalzahlen.
Die Kloostermansche Methode läßt sich etwas vereinfachen, wenn man sie mit der Streefkerkschen kombiniert und Thetafunktionen mit Charakteristiken, statt der üblichen Jacobischen Funktionen, anwendet. Diese kombinierte Methode erfordert auch lange Rechnungen, die aber rein elementar sind.

Auf diese Weise werden in der vorliegenden Arbeit exakte Formeln für $r(n): a=1, a^{\prime}=5,6,7,9,10 ; a=2, a^{\prime}=5$ gegeben. In den Fällen $a=1 ; a^{\prime}=5,6,7$ stimmen sie mit den Kloostermanschen überein. Die Formel für $a=1, a^{\prime}=9, n \neq 1(\bmod 6)$ war schon von Liouville

[6] rein arithmetisch erhalten und ohne Beweis veröffentlicht worden. Mit dem in der vorliegenden Arbeit benutzten Verfahren können ana$\log$ auch andere Gleichungen (2.1) behandelt werden.

Im folgenden soll, ohne Beschränkung der Allgemeinheit,

$$
\left(a, a^{\prime}\right)=1, \quad a^{\prime} \equiv 1(\bmod 2)
$$

angenommen werden.

§3. In diesem Paragraphen werden ohne Beweis einige bekannte Ergebnisse angeführt.

Es sei $\Gamma$ die Modulgruppe, d. h. die Gruppe aller linearen Substitutionen

wo $\alpha \delta-\beta \gamma=1$.

$$
\tau^{\prime}=\frac{\alpha \tau+\beta}{\gamma \tau+\delta}
$$
durch

Weiter sei $\Gamma(N)$ die Hauptkongruenzgruppe der Stufe $N$, d. h. die

$$
\alpha \equiv \delta \equiv 1(\bmod N), \quad \beta \equiv \gamma \equiv 0(\bmod N)
$$

bestimmte Untergruppe von $\Gamma$.

Defantrion 1 (vgl. z. B. [3], S. 167). Eine analytische Funktion $F(\tau)$ heißt eine ganze Modulform der Stufe $N$ und der Dimension $-r$, falls sie den folgenden Bedingungen genügt:

1. sie ist regulär und eindeutig in der Halbebene $\operatorname{Im} \tau>0$;

2. für jede Substitution der Gruppe $\Gamma(N)$ gilt

$$
F\left(\frac{\alpha \tau+\beta}{\gamma \tau+\delta}\right)=(\gamma \tau+\delta)^{r} F(\tau)
$$

3. in der Umgebung vom $\tau=\infty$ gilt eine Potenzreihenentwicklung

$$
F(\tau)=C_{0}+\sum_{n=1}^{\infty} C_{n} e\left(\frac{n \tau}{N}\right)
$$


4. in der Umgebung von

$$
\tau=-\frac{\delta}{\gamma} \quad(\gamma \neq 0,(\gamma, \delta)=1, \alpha \delta-\beta \gamma=1)
$$

gilt eine Potenzreihenentwicklung

$$
(\gamma \tau+\delta)^{r} F(\tau)=C_{0}^{\prime}+\sum_{n=1}^{\infty} C_{n}^{\prime} e\left(\frac{n}{N} \cdot \frac{\alpha \tau+\beta}{\gamma \tau+\delta}\right) .
$$

Ist im letzten Falle $C_{0}^{\prime}=C_{1}^{\prime}=\ldots=C_{m-1}^{\prime}=0(m \geqslant 1)$, so wollen wir sagen, daß $F^{\prime}(\tau)$ in $\tau=-\delta / \gamma$ eine $m$-fache Nullstelle besitzt.

HIlfFssatz 1 (vgl. z. B. [1]; I, S. 397 und II, S. 363). Die ganze Modulform der Stufe $N$ und der Dimension $-r$ ist identisch $N$ ull, wenn sie im Fundamentalbereich mehr als

$$
\frac{r}{24} N^{3} \prod_{p \mid N}\left(1-p^{-2}\right)
$$

Nullpunkte (mehrfache mehrfach gezählt) besitzt.

HILFSSATZ 2. Fiür $(h, q)=1$ ist

$$
S(a h, a q)=a S(h, \dot{q}) \text {. }
$$

Dies folgt unmittelbar aus (1.2).

Hilfssatz 3 (vgl. z. B. [9], S. 13, 'Hilfssatz 6). Es sei $(h, q)=1$. Dann ist

$$
S^{2}(h, q)=\left\{\begin{array}{cll}
\left(\frac{-1}{q}\right) q & \text { für } & q \equiv 1(\bmod 2), \\
0 & \text { für } & q \equiv 2(\bmod 4), \\
2 i^{h} q & \text { für } & q \equiv 0(\bmod 4) .
\end{array}\right.
$$

HILFSSATZ 4 ([2], S. 149, Formel 2.413). Frür $H=h q^{\prime}+h^{\prime} q$, $(h, q)=\left(h^{\prime}, q^{\prime}\right)=\left(q, q^{\prime}\right)=1$ ist

$$
S\left(a H, q q^{\prime}\right)=S\left(a h^{\prime}, q^{\prime}\right) S(a h, q) \text {. }
$$

HILFSSATZ 5 (vgl. z. B. [2], S. 146, Formel 2.123). Für $\left(q, q^{\prime}\right)=1$

$$
\iota\left(h, q q^{\prime}\right)=c(h, q) c\left(h, q^{\prime}\right) .
$$

Huffssatz 6 (vgl. z. B. [2], S. 146, Formel 2.124).

$$
c(h, q)=\sum_{d \mid(h, q)} d \mu\left(\frac{q}{d}\right) .
$$

Hillassatz 7 (vgl. z. B. [2], S. 146, Formel 2.125). Es sei $q=p^{\lambda}$ $(\lambda \geqslant 1)$ und $p^{x} \| h$. Dann. ist

$$
c(h, q)=\left\{\begin{array}{lll}
0 & \text { für } & x<\lambda-1, \\
-p^{\lambda-1} & \text { für } & x=\lambda-1, \\
p^{\lambda-1}(p-1) & \text { für. } & x>\lambda-1 .
\end{array}\right.
$$

HILFSSATz 8 ([8], S. 29, Hilfssatz 28). Für $\varrho>1, \operatorname{Im} \tau>0$ und $0<\arg (\tau N-n)<\pi$ ist

$$
\sum_{\substack{M=1 \\ M \equiv c(\bmod N)}}^{\infty} M^{\rho-1} e(M \tau)=(2 \pi)^{-\varrho} N^{\varrho-1} \Gamma(\varrho) e\left(\frac{\varrho}{4}\right) \sum_{n=-\infty}^{\infty} \frac{e(n c / N)}{(\tau N-n)^{\varrho}} .
$$

HiLfssatz 9 ([8], S. 29, Hilfssatz 29). Es sei $\varrho>1,0<\xi<1, v>0$. Dann ist für $\xi \rightarrow 1$

$$
\sum_{\substack{M=1 \\ M=c(\bmod )}}^{\infty} M^{e-1} \xi^{y M} \sim y^{-\varrho} N^{-1} \Gamma(\varrho)\left(\log \frac{1}{\xi}\right)^{-\varrho}
$$

Hrufssatz 10 ([8], S. 44, Hilfssatz 47). Es sei $0<\xi<1, \nu>0$. Dann ist für $\xi \rightarrow 1$

$$
\sum_{j \equiv h(\bmod q)} \xi^{\eta j^{2}} \sim \frac{\sqrt{\pi}}{q \sqrt{\nu}}\left(\log \frac{1}{\xi}\right)^{-1 / 2} .
$$

Hilfssatz 11 (vgl. z. B. [9], S. 126, Hilfssatz 1). Die stetige Funkl tion $f(t)$ sei im Intervall $0 \leqslant t<\infty$ definiert, ihr Real- und Imaginärteidort von beschränkter Variation. Außerdem sei $\lim f(t)=0$ und das Integral $\int_{0}^{\infty} f(t) d t$ vorhanden. Dann ist

$$
\int_{0}^{\infty} f(t) d t+2 \sum_{m=1}^{\infty} \int_{0}^{\infty} f(t) \cos 2 m \pi t d t=\frac{1}{2} f(0)+\sum_{m=1}^{\infty} f(m) .
$$

HILfSSATz 12 (vgl. z. B. [8], S. 31, Hilfssatz 31). Das Integral

$$
U(z ; \zeta, \tau, v)=\int_{-\infty}^{\infty} \frac{e(-\nu w) d w}{(w+\tau)^{\zeta}|w+\tau|^{z}}
$$

konvergiert absolut für $\operatorname{Re}(z+\zeta)>1$. Die Funktion $U(z ; \zeta, \tau, v)$ besitzt folgende Eigenschaften:

1. für $\nu \neq 0$ kann sie in die ganze $z$-Ebene fortgesetzt werden;

2. $U(z ; \zeta, \tau, 0)$ ist in der Halbebene $\operatorname{Re}(z+\zeta)>1$ regulär; 
3. ist $D$ ein beschränltes Gebiet der z-Ebene und $v \neq 0$, so gilt eine Ungleichung der. Gestalt

$$
|U(z ; \zeta, \tau, v)|<K e^{-K^{\prime}|v|},
$$

wobei $K$ und $K^{\prime}$ von $v$ und $z$ unabhängig sind; falls $v=0$, gilt diese Ungleichung auch für $\operatorname{Re} z>1-\operatorname{Re} \zeta$;

$$
\text { 4. } U(0 ; \zeta, \tau, \nu)= \begin{cases}0 & \text { für } v<0, \\ \Gamma^{-1}(\zeta) e\left(-\frac{\zeta}{4}\right)(2 \pi)^{\zeta} \nu^{\zeta-1} e(\tau \nu) & \text { für } v>0, \zeta \neq 0, \\ & -1,-2, \ldots,\end{cases}
$$$$
U(0 ; \zeta, \tau, 0)=0 \text { für } \operatorname{Re} \zeta>1 \text { und für } \zeta=1 \text {. }
$$

§ 4. In diesem Paragraphen soll die zu $r(n)$ gehörige singuläre Reihe formal konstruiert werden, wobei gezeigt wird, daß sie absolut konvergiert.

Sei

$$
M=4 a a^{\prime} n
$$

Aus (2.1) folgt dann

$$
M=\sum_{\substack{k=1 \\ y_{k} \equiv 0(\bmod 2 a)}}^{2} a^{\prime} y_{k}^{2}+\sum_{\substack{k=3 \\ y_{k} \equiv 0\left(\bmod 2 a^{\prime}\right)}}^{4} a y_{k}^{2} .
$$

$R(M)$ bezeichne die Lösungszahl der Gleichung (4.1). Offenbar ist

$$
r(n)=R(M) .
$$

Aus (1.4), (1.5) und (4.1) folgt

$$
\vartheta_{00}^{2}(\tau ; 0,2 a) \vartheta_{00}^{2}\left(\tau ; 0,2 a^{\prime}\right)=1+\sum_{\substack{M=1 \\ M=0\left(\bmod 4 a a^{\prime}\right)}}^{\infty} R(M) Q^{M}
$$

Es soll jetzt das Verhalten von $\vartheta_{00}^{2}(\tau ; 0,2 a) \vartheta_{00}^{2}\left(\tau ; 0,2 \alpha^{\prime}\right)$ bei Annäherung von $\tau$ an die rationale Stelle $h / q((h, q)=1)$ untersucht werden. $\mathrm{Zu}$ dem Zwecke setze man

$$
e(\tau)=W e\left(\frac{h}{q}\right), \quad 0<W<1
$$

und lasse $W$ gegen 1 rücken.
Nach (1.4), (1.5), (4.4), (1.2) und Hilfssatz 10 ist

$$
\begin{aligned}
\vartheta_{00}(\tau ; 0,2 a) & =\sum_{\substack{y=-\infty \\
y=0(\bmod 2 a)}}^{\infty} W^{v^{2} / 4 a} e\left(\frac{y^{2} h}{4 a q}\right) \\
& =\sum_{\substack{y \bmod 2 \alpha q \\
y \equiv 0(\bmod 2 a)}} e\left(\frac{y^{2} h}{4 a q}\right) \sum_{j \equiv y(\bmod 2 a q)} W^{j^{2} / 4 a} \sim \frac{\sqrt{\pi}}{\sqrt{a} q} s(a h, q)\left(\log \frac{1}{W}\right)^{-1 / 2} ;
\end{aligned}
$$

analog ist

$$
\vartheta_{00}\left(\tau ; 0,2 a^{\prime}\right) \sim \frac{\sqrt{\pi}}{\sqrt{a^{\prime}} q} S\left(a^{\prime} h, q\right)\left(\log \frac{1}{W}\right)^{-1 / 2} .
$$

Setzt man also zur Abkürzung

so folgt

$$
\tilde{S}(h, q)=S(a h, q) S\left(a^{\prime} h, q\right),
$$

(4.5) $\quad \vartheta_{00}^{2}(\tau ; 0,2 a) \vartheta_{00}^{2}\left(\tau ; 0,2 a^{\prime}\right) \sim \frac{\pi^{2}}{a a^{\prime} q^{4}} \tilde{S}^{2}(h, q)\left(\log \frac{1}{W}\right)^{-2}$.

Aus (4.5), (4.4) und Hilfssatz 9 ergibt sich

(4.6) $\vartheta_{00}^{2}(\tau ; 0,2 a) \vartheta_{00}^{2}\left(\tau ; 0,2 a^{\prime}\right) \sim \frac{\pi^{2}}{4 a^{2} a^{\prime 2} q^{4}} \tilde{S}^{2}(h, q) \sum_{\substack{M=1 \\ M=0\left(\bmod 4 a a^{\prime}\right)}}^{\infty} M e\left(-\frac{h M}{4 a a^{\prime} q}\right) Q^{M}$

Es sei

$$
\Theta(\tau)=\Theta\left(\tau ; a a^{\prime}\right)=1+\sum_{\substack{M=1 \\ M=0\left(\bmod 4 a a^{\prime}\right)}}^{\infty} \varrho(M) Q^{M},
$$

wo $\varrho(M)$ wie folgt definiert ist:

$$
\begin{gathered}
\varrho(M)=\frac{\pi^{2}}{4 a^{2} a^{\prime 2}} M \sum_{q=1}^{\infty} A_{q}, \\
A_{q}=q^{-4} \sum_{h \bmod q}^{Y^{\prime}} e\left(-\frac{h M}{4 a a^{\prime} q}\right) \tilde{S}^{2}(h, q) .
\end{gathered}
$$

Nach (4.6) - (4.9) verhalten sich die Funktionen $\vartheta_{00}^{2}(\tau ; 0,2 a) \vartheta_{00}^{2}\left(\tau ; 0,2 a^{\prime}\right)$ und $\Theta(\tau)$ identisch bei Annäherung an rationale Randpunkte.

Die Reihe (4.8) ist die gesuchte singuläre Reihe. 
HrLfssatz 13. Die Reihe $\sum_{q=1}^{\infty} A_{q}$ konvergiert absolut.

Beweis. Es sei

$(h, q)=1, \quad q=(q, a) q_{1}, \quad a=(q, a) a_{1}, \quad q=\left(q, a^{\prime}\right) q_{1}^{\prime}, \quad a^{\prime}=\left(q, a^{\prime}\right) a_{1}^{\prime}$.

Nach Hilfssatz 2 ist

(4.10) $S(a h, q)=(q, a) S\left(a_{1} h, q_{1}\right), \quad S\left(a^{\prime} h, q\right)=\left(q, a^{\prime}\right) S\left(a_{1}^{\prime} h, q_{1}^{\prime}\right)$.

Aus (4.10), (2.2) und Hilfssatz 3 ergibt sich

(4.11) $\quad \tilde{S}^{2}(h, q)=\left\{\begin{array}{l}\left(\frac{-1}{q_{1} q_{1}^{\prime}}\right)(q, a)\left(q, a^{\prime}\right) q^{2} \\ 0, \quad \text { wenn mindestens eine der beiden Bedingungen }\end{array}\right.$ $2\left(\frac{-1}{q_{1}}\right)(q, a)\left(q, a^{\prime}\right) q^{2} e\left(\frac{1}{4} h a_{1}^{\prime}\right)$ für $2 \times q_{1}, q_{1}^{\prime} \equiv 0(\bmod 4)$,

$4(q, a)\left(q, a^{\prime}\right) q^{2} e\left(\frac{1}{4} h a_{1}+\frac{1}{4} h a_{1}^{\prime}\right)$ für $q_{1} \equiv q_{1}^{\prime} \equiv 0(\bmod 4)$.

Nach (4.9) und (4.11) ist

$\left|A_{q}\right|<K q^{-2}\left|c\left(\frac{M}{4 a a^{\prime}}, q\right)\right| \quad$ für $\quad 2 \times q$

$A_{q}=0$, wenn mindestens eine der beiden Bedingungen

$q_{1} \equiv 2(\bmod 4), q_{1}^{\prime} \equiv 2(\bmod 4)$ erfüllt ist,

$\left|A_{q}\right|<K q^{-2}\left|e\left(\frac{M-a a^{\prime} a_{1}^{\prime} q}{4 a a^{\prime}}, q\right)\right| . \quad$ für $\quad 2 \times q_{1}, q_{1}^{\prime} \equiv 0(\bmod 4)$,

$\left|A_{q}\right|<K q^{-2}\left|c\left(\frac{1}{4 a a^{\prime}}\left(M-a_{1} a a^{\prime} q-a_{1}^{\prime} a a^{\prime} q\right), q\right)\right|$ für $q_{1} \equiv q_{1}^{\prime} \equiv 0(\bmod 4)$,

wo $K$ nicht von $q$ abhängt. Nach Hilfssatz 6 ist also

$$
\left|A_{q}\right|<K q^{-2} \sum_{d \mid M} d
$$

woraus die Behauptung folgt.

§ 5. In diesem Paragraphen sollen gewisse Eigenschaften der Thetafunktionen mit Charakteristiken hergeleitet werden.
Bekanntlich ist (vgl. z. B. [4], S. 318-319, Formeln (1.2)-(1.5))

$$
\begin{gathered}
\vartheta_{g, h+2 k}(z \mid \tau ; c, N)=\vartheta_{g h}(z \mid \tau ; c, N), \\
\vartheta_{g+2 k, h}(z \mid \tau ; c, N)=\vartheta_{g h}(z \mid \tau ; c+k, N), \\
\vartheta_{g h}(z \mid \tau ; c+N k, N)=(-1)^{h k} \vartheta_{g h}(z \mid \tau ; c, N), \\
\vartheta_{g h}\left(z+\frac{1}{2 N}(l+m \tau) \mid \tau ; c, N\right) \\
=e\left(\frac{1}{2 N}\left(c+\frac{1}{2} g\right) l\right) e\left(-\frac{1}{8 N} m^{2} \tau\right) e\left(-\frac{1}{2} m z\right) \vartheta_{g+m, h+l}(z \mid \tau ; c, N) .
\end{gathered}
$$

Hufssatz 14. Die Identität

$$
\vartheta_{g h}(\tau ; c, N)=0
$$

gilt dann und nur dann, wenn

$$
g \equiv N-2 c(\bmod 2 N), \quad h \equiv 1(\bmod 2) .
$$

Beweis. Setzt man in (5.4): $l=2, m=0$, so ergibt sich wegen (5.1)

$$
\vartheta_{g h}\left(z+\frac{1}{N} \mid \tau ; c, N\right)=e\left(\frac{1}{N}\left(c+\frac{1}{2} g\right)\right) \vartheta_{g h}(z \mid \tau ; c, N) ;
$$

für $l=0, m=2 N$ folgt aus (5.2) und (5.3)

(5.6) $\vartheta_{g h}(z+\tau \mid \tau ; c, N)=e\left(-\frac{1}{2} N \tau\right) e(-N z)(-1)^{h} \vartheta_{g h}(z \mid \tau ; c, N)$.

Differenziert man (5.5) und (5.6) logarithmisch nach $z$, so bekommt $\operatorname{man}$

und

$$
\frac{\vartheta_{g h}^{\prime}\left(z+\frac{1}{N} \mid \tau ; c, N\right)}{\vartheta_{g h}\left(z+\frac{1}{N} \mid \tau ; c, N\right)}=\frac{\vartheta_{g h}(z \mid \tau ; c, N)}{\vartheta_{g h}(z \mid \tau ; c, N)}
$$

$$
\frac{\vartheta_{g h}^{\prime}(z+\tau \mid \tau ; c, N)}{\vartheta_{g h}(z+\tau \mid \tau ; c, N)}=-2 N \pi i+\frac{\vartheta_{g h}^{\prime}(z \mid \tau ; c, N)}{\vartheta_{g h}(z \mid \tau ; c, N)} .
$$

Es sei $L$ ein Parallelogramm mit den Ecken

$$
\zeta, \zeta+\frac{1}{N}, \zeta+\frac{1}{N}+\tau, \zeta+\tau .
$$


Dann ist die Anzahl der Nullpunkte von $\vartheta_{g h}(z \mid \tau ; c, N)$ im Innern von $L$ gleich

$$
\begin{aligned}
\frac{1}{2 \pi i} \int_{L} \frac{\vartheta_{g h}^{\prime}(z \mid \tau ; c, N)}{\vartheta_{g h}(z \mid \tau ; c, N)} d z & \\
= & \frac{1}{2 \pi i} \int_{\zeta}^{\zeta+1 / N}\left\{\frac{\vartheta_{g h}^{\prime}(z \mid \tau ; o, N)}{\vartheta_{g h}(z \mid \tau ; o, N)}-\frac{\vartheta_{g h}^{\prime}(z+\tau \mid \tau ; c, N}{\vartheta_{g h}(z+\tau \mid \tau ; c, N)}\right\} d z- \\
& -\frac{1}{2 \pi i} \int_{\zeta}^{\zeta+\tau}\left\{\frac{\vartheta_{g h}^{\prime}(z \mid \tau ; c, N)}{\vartheta_{g h}(z \mid \tau ; c, N)}-\frac{\vartheta_{g h}^{\prime}\left(z+\frac{1}{N} \mid \tau ; c, N\right)}{\vartheta_{g h}\left(z+\frac{1}{N} \mid \tau ; c, N\right)}\right\} d z=1,
\end{aligned}
$$

nach (5.7) und (5.8).

Aus (1.5) und (1.4) folgt

$$
\vartheta_{N-2 c, 1}(\tau ; c, N)=\sum_{k=-\infty}^{\infty}(-1)^{k} e\left(\frac{1}{8}(2 k+1)^{2} N \tau\right)=0 .
$$

Setzt man also in (5.4): $z=0, l=1-h, m=N-2 c-g$, so ergibt sich

$$
\begin{aligned}
& \vartheta_{g h}\left(\frac{1}{2 N}(1-h+(N-2 c-g) \tau) \mid \tau ; c, N\right) \\
= & e\left(\frac{1}{2 N}\left(c+\frac{1}{2} g\right)(1-h)\right) e\left(-\frac{1}{8 N}(N-2 e-g)^{2} \tau\right) \vartheta_{0+(N-2 c-g), h+(1-h)}(\tau ; c, N)=0 .
\end{aligned}
$$

Daher ist, nach dem Obigen,

$$
z=\frac{1}{2 N}(1-h)+\frac{1}{2 N}(N-2 c-g) \tau
$$

die einzige Nullstelle von $\vartheta_{g h}(z \mid \tau ; c, N)$ im Innern von $L$. Hieraus folgt nach (5.1)-(5.3) und (5.5), (5.6) die Behauptung.

HrLfssatz 15 ([7], S. 84, Hilfssatz 7). Bei geradem g gilt für jede Substitution der Gruppe $\Gamma(2 N)$

$$
\begin{aligned}
& \vartheta_{g h}\left(\frac{\alpha \tau+\beta}{\gamma \tau+\delta} ; 0, N\right) \\
& \quad=e\left(-\frac{\alpha \gamma \delta^{2} h^{2}}{8 N}\right) i^{1 / 2 \operatorname{sgn} \gamma(\operatorname{sgn} \delta-1)} \frac{\sqrt{\gamma \tau+\delta}}{\sqrt{|\delta|}} S\left(\frac{1}{2} \beta N \operatorname{sgn} \delta,|\delta|\right) \vartheta_{g h}(\tau ; 0, N) .
\end{aligned}
$$

Hulfssatz 16 ([7], S. 86, Hilfssatz 8). Es seien $g$ und $N$ gerade, $a \delta-\beta \gamma=1$. Dann gilt in der Umgebung von

$$
\tau=-\frac{\delta}{\gamma} \quad(\gamma \neq 0,(\gamma, \delta)=1)
$$

die Entwicklung

$$
\sqrt{\gamma \tau+\delta} \vartheta_{g h}(\tau ; c, N)=\left\{\begin{array}{l}
\sum_{n=0}^{\infty} B_{n} e\left(\frac{n}{2 N} \cdot \frac{\alpha \tau+\beta}{\gamma \tau+\delta}\right) \quad \text { für gerade } \quad \gamma h, \\
e\left(\frac{1}{8 N} \cdot \frac{\alpha \tau+\beta}{\gamma \tau+\delta}\right) \sum_{n=0}^{\infty} B_{n}^{\prime} \epsilon\left(\frac{n}{2 N} \cdot \frac{\alpha \tau+\beta}{\gamma \tau+\delta}\right) \text { für ungerade } \gamma h .
\end{array}\right.
$$

Hilfssatz 17. Die Funktion $\vartheta_{00}^{2}(\tau ; 0,2 a) \vartheta_{00}^{2}\left(\tau ; 0,2 a^{\prime}\right)$ ist eine ganze Modulform der Stufe $4 a a^{\prime}$ und der Dimension -2 .

Beweis. Nach (1.5) und (4.3) erfüllt die Funktion $\vartheta_{00}^{2}(\tau ; 0,2 a) \vartheta_{00}^{2}(\tau$; $0,2 a^{\prime}$ ) die Bedingungen 1 und 3 von Definition 1 .

Man beachte, daß jede Substitution der Gruppe $\Gamma\left(4 a a^{\prime}\right)$ auch eine Substitution der Gruppen $\Gamma(4 a)$ und $\Gamma\left(4 a^{\prime}\right)$ ist. Daher gilt nach den Hilfssätzen 15 und 3 für jede Substitution der Gruppe $\Gamma\left(4 a a^{\prime}\right)$

$$
(\gamma \tau+\delta)^{-2} \vartheta_{00}^{2}\left(\frac{\alpha \tau+\beta}{\gamma \tau+\delta} ; 0,2 a\right) \vartheta_{00}^{2}\left(\frac{\alpha \tau+\beta}{\gamma \tau+\delta} ; 0,2 a^{\prime}\right)=\vartheta_{00}^{2}(\tau ; 0,2 a) \vartheta_{00}^{2}\left(\tau ; 0,2 \boldsymbol{a}^{\prime}\right) \text {. }
$$

Die Bedingung 2 von Definition 1 ist also erfüllt.

Nach Hilfssatz 16 gilt in der Umgebung von

$$
\tau=-\frac{\delta}{\gamma} \quad(\gamma \neq 0,(\gamma, \delta)=1, \alpha \delta-\beta \gamma=1)
$$

die Entwicklung

$$
(\gamma \tau+\delta)^{2} \vartheta_{00}^{2}(\tau ; 0,2 a) \vartheta_{00}^{2}\left(\tau ; 0,2 \alpha^{\prime}\right)=\sum_{n=0}^{\infty} D_{n} e\left(\frac{n}{4 a a^{\prime}} \cdot \frac{a \tau+\beta}{\gamma \tau+\delta}\right) .
$$

Die Bedingung 4 von Definition 1 ist also auch erfüllt.

§6. In diesem Paragraphen wird mittels der Streefkerkschen Methode gezeigt, daß die durch (4.7)-(4.9) definierte Funktion $\Theta(\tau)$ eine ganze Modulform ist.

Wir führen die folgende Hilfsfunktion ein:

$$
\Theta(\tau, z)=1-\frac{1}{8 a a^{\prime}} \sum_{\substack{q, H=-\infty \\(H, q)=1}}^{\infty}\left(\frac{\tilde{S}(-H \operatorname{sgn} q,|q|}{q(q \tau+H)}\right)^{2}|q \tau+H|^{-z}
$$


wo der Strich bedeutet, daß die Glieder mit $q=0$ auszuschließen sind. Nach Hilfssatz 3 ist $\Theta(\tau, z)$ bei festem $\tau$ für $\operatorname{Re} z>0$ regulär.

HILFSSATZ 18. Es sei

(6.2) $T(m, z)=\int_{-\infty}^{\infty} e(-m t)\left(\frac{\tau}{4 a a^{\prime}}+t\right)^{-2}\left|\frac{\tau}{4 a a^{\prime}}+t\right|^{-z} d t$,

(6.3) $\quad F(m, z) \doteq 1+\sum_{\lambda=1}^{\infty} 2^{-\lambda(1+z)} \sum_{h \bmod 2^{\lambda}}^{\prime} e\left(-\frac{m h}{2^{\lambda+2} a a^{\prime}}\right) \tilde{S}^{2}\left(h, 2^{\lambda}\right)$,

(6.4) $\quad G(m, z)=\sum_{\substack{q=1 \\(q, 2)=1}}^{\infty} q^{-4-z} \sum_{x \bmod q}^{\prime} e\left(-\frac{m x}{4 a a^{\prime} q}\right) \tilde{S}^{2}(x, q)$.

Dann ist für Re $z>0$

(6.5) $\Theta(\tau, z)=1-2^{-4-2 z}\left(a a^{\prime}\right)^{-2-z} \sum_{\substack{m=-\infty \\ m \equiv 0\left(\bmod 4 a a^{\prime}\right)}}^{\infty} T(m, z) F(m, z) G(m, z)$.

Beweis. Für Re $z>0$ folgt aus (6.1)

$$
\Theta(\tau, z)=1-\frac{1}{4 a a^{\prime}} \sum_{q=1}^{\infty} \sum_{\substack{H=-\infty \\(H, q)=1}}^{\infty}\left(\frac{\tilde{S}(-H, q)}{q(q \tau+H)}\right)^{2}|q \tau+H|^{-z}
$$

Setzt man $H=l+4 a a^{\prime} q m$, so ergibt sich

(6.6) $\Theta(\tau, z)=1-\left(4 a a^{\prime}\right)^{-3-z} \sum_{q=1}^{\infty} q^{-4-z} \sum_{\substack{l \bmod (l, q)=1 \\(l, \alpha)}} \tilde{S}^{2}(-l, q) \times$

$$
\times \sum_{m=-\infty}^{\infty}\left(\frac{\tau}{4 a a^{\prime}}+\frac{l}{4 a a^{\prime} q}+m\right)^{-2}\left|\frac{\tau}{4 a a^{\prime}}+\frac{l}{4 a a^{\prime} q}+m\right|^{-2}
$$

Nach Hilfssatz 11 und (6.2) ist, falls $t=w+l / 4 a a^{\prime} q$ gesetzt wird,

$$
\sum_{m=-\infty}^{\infty}\left(\frac{\tau}{4 a a^{\prime}}+\frac{l}{4 a a^{\prime} q}+m\right)^{-2}\left|\frac{\tau}{4 a a^{\prime}}+\frac{l}{4 a a^{\prime} q}+m\right|^{-\approx}
$$

$=\sum_{m=-\infty}^{\infty} \int_{-\infty}^{\infty}\left(\frac{\tau}{4 a a^{\prime}}+\frac{l}{4 a a^{\prime} q}+w\right)^{-2}\left|\frac{\tau}{4 a a^{\prime}}+\frac{l}{4 a a^{\prime} q}+w\right|^{-z} e(-m w) d w$

$$
=\sum_{m=-\infty}^{\infty} e\left(\frac{m l}{4 a a^{\prime} q}\right) T(m, z)
$$

Wegen $\operatorname{Re} z>0$ folgt aus (6.6) und (6.7)

(6.8) $\Theta(\tau, z)=$

$$
1-\left(4 a a^{\prime}\right)^{-3-z} \sum_{m=-\infty}^{\infty} T(m, z) \sum_{q=1}^{\infty} q^{-4-z} \sum_{\substack{l \text { mod } q a a^{\prime} q \\(l, q)=1}} e\left(\frac{m l}{4 a a^{\prime} q}\right) \tilde{s}^{2}(-l, q) .
$$

Für $l=k+j q$ ergibt sich daraus

$$
\begin{aligned}
\sum_{\substack{l \bmod 4 a a^{\prime} q \\
(l, q)=1}} e\left(\frac{m l}{4 a a^{\prime} q}\right) \tilde{S}^{2}(-l, q) & =\sum_{\substack{k=1 \\
(k, q)=1}}^{q} e\left(\frac{m k}{4 a a^{\prime} q}\right) \tilde{S}^{2}(-k, q) \sum_{j=0}^{4 a a^{\prime}-1} e\left(\frac{m j}{4 a a^{\prime}}\right) \\
& = \begin{cases}4 a a^{\prime} \sum_{k \bmod q}^{\prime} e\left(-\frac{m k}{4 a a^{\prime} q}\right) \tilde{S}^{2}(k, q) & \text { für } 4 a a^{\prime} \mid m, \\
0 & \text { für } 4 a a^{\prime} \times m .\end{cases}
\end{aligned}
$$

Aus (6.8) und (6.9) folgt also

$$
\Theta(\tau, z)=1-\left(4 a a^{\prime}\right)^{-2-z} \sum_{\substack{m=-\infty \\ m=0\left(\bmod 4 a a^{\prime}\right)}}^{\infty} T(m, z) \sum_{q=1}^{\infty} q^{-4-z} \sum_{k \bmod q}^{\prime} e\left(-\frac{m k}{4 a a^{\prime} q}\right) \tilde{s}^{2}(k, q) .
$$

Setzt man hier $q=2^{\lambda} q^{\prime}\left(q^{\prime}\right.$ ungerade, $\left.\lambda \geqslant 0\right) \quad k=x \cdot 2^{\lambda}+h q^{\prime} \quad(\lambda \geqslant 1)$, berücksichtigt Hilfssatz 4 und schreibt $q$ statt $q^{\prime}$, so ergibt sich die Behauptung.

Hufssatz 19. Die durch (6.3) definierte Funktion $F(m, z)$ ist eine ganze Funktion von z. In der Halbebene $\operatorname{Re} z>-1$ gilt

$$
|F(m, z)|<K|m|^{s}, \quad \varepsilon>0,
$$

wo $K$ nicht von $m$ und $z$ abhängt. folgt

Beweis. Es sei $a=2^{\gamma} b(\gamma \geqslant 0, b$ ungerade). Aus (6.3) und Hilfssatz 3

(6.10) $\quad F(m, z)=$

$$
1+\sum_{\lambda=2}^{\infty} 2^{-\lambda(4+z)} \sum_{h \bmod 2^{\lambda}}^{\prime} e\left(-\frac{m h}{2^{\lambda+2+\gamma} b a^{\prime}}\right) S^{2}\left(2^{\gamma} b h, 2^{\lambda}\right) S^{2}\left(a^{\prime} h, 2^{\lambda}\right) .
$$

Führt man jetzt einen neuen Summationsbuchstaben $k$ durch $h \equiv b a^{\prime} k\left(\bmod 2^{\lambda}\right)$ ein, so ergibt sich nach den Hilfssätzen 2 und 3 
(6.11) $\quad H^{\prime}(m, z)$

$$
\begin{aligned}
= & 1+\sum_{\lambda=2}^{\gamma} 2^{-\lambda(4+z)} \sum_{k \bmod 2^{\lambda}}^{\prime} e\left(-\frac{m k}{2^{\lambda+\gamma+2}}\right) 2^{2 \lambda} S^{2}\left(b a^{\prime 2} k, 2^{\lambda}\right)+ \\
& +\sum_{\lambda=\gamma+2}^{\infty} 2^{-\lambda(4+z)} \sum_{k \bmod 2^{\lambda}}^{\prime} e\left(-\frac{m k}{2^{\lambda+\gamma+2}}\right) 2^{2 \gamma} S^{2}\left(b^{2} a^{\prime} k, 2^{\lambda-\gamma}\right) S^{2}\left(b a^{\prime 2} k, 2^{\lambda}\right) \\
= & 1+\sum_{\lambda=2}^{\gamma} 2^{1-\lambda(1+z)} c\left(\frac{2^{\lambda+\gamma} b-m}{2^{\gamma+2}}, 2^{\lambda}\right)+ \\
& +\sum_{\lambda=\gamma+2}^{\infty} 2^{\gamma+2-\lambda(2+z)}(-1)^{\left(b+a^{\prime}\right) / 2} c\left(\frac{m}{2^{\gamma+2}}, 2^{\lambda}\right) .
\end{aligned}
$$

Es sei $2^{x} \| m(x \geqslant \gamma+2)$, d. h. $2^{x-\gamma-2} \| \frac{m}{2^{\gamma+2}}$. Nach Hilfssatz 7 ist dann

$$
o\left(\frac{m}{2^{\gamma+2}}, 2^{\lambda}\right)=0 \quad \text { für } \quad x-\gamma-2<\lambda-1 .
$$

Aus (6.11) folgt also, daß $F(m, z)$ eine endliche summe von in jedem endlichen Gebiet regulären Funktionen ist.

Ferner ist

$$
\begin{aligned}
\left|F^{\prime}(m, z)\right| & \leqslant 1+\sum_{\lambda=2}^{\gamma}\left|2^{1-\lambda(1+z)}\right| \cdot 2^{\lambda}+\sum_{\lambda=\gamma+2}^{\gamma-\gamma-1}\left|2^{\gamma+2-\lambda(2+z)}\right| \cdot 2^{\lambda} \\
& <1+\sum_{\lambda=2}^{x-\gamma-1} 2^{\gamma+2-\lambda(\mathrm{R} \theta z+1)}
\end{aligned}
$$

folglich für $\operatorname{Re} z>-1$

$$
|F(m, z)|<1+\varkappa \cdot 2^{\gamma+2}<K|m|^{\varepsilon}, \quad \varepsilon>0 .
$$

HiLFsSatz 20. Die durch $\{6.4)$ definierte Funktion $G(m, z)$ ist in der Halbebene $\operatorname{Re} z>-\frac{1}{2}$ in $z$ regulär und dort

$$
|G(m, z)|<K m^{2},
$$

wobei $K$ nicht von $m$ und $z$ abhängt.

Beweis. Aus (6.4) und (4.11) folgt

$$
G(m, z)=\sum_{\substack{q=1 \\(q, 2)=1}}^{\infty} A_{q}(z),
$$

wo

$$
\begin{gathered}
A_{q}(z)=\left(\frac{-1}{q_{1} q_{1}^{\prime}}\right)(q, a)\left(q, a^{\prime}\right) q^{-2-z} c\left(\frac{m}{4 a a^{\prime}}, q\right), \\
q_{1}=\frac{q}{(q, a)}, \quad q_{1}^{\prime}=\frac{q}{\left(q, a^{\prime}\right)} .
\end{gathered}
$$

Nach Hilfssatz 6 ist

$$
\left|A_{q}(z)\right| \leqslant a a^{\prime} q^{-2-\operatorname{Re} z} \sum_{d_{l}\left(m / 4 a a^{\prime}, q\right)} d \leqslant a a^{\prime} q^{-2-\operatorname{Re} z} m^{2},
$$

und daher für $\operatorname{Re} z>-\frac{1}{2}$

$$
\left|A_{q}(z)\right|<a a^{\prime} m^{2} q^{-3 / 2} .
$$

Hieraus ergibt sich, daß die Reihe

$$
\sum_{\substack{q=1 \\(q, 2)=1}}^{\infty} A_{q}(z)
$$

in der Halbebene Re $z>-\frac{1}{2}$ absolut und gleichmäßig konvergiert. Außerdem ist für $\operatorname{Re} z>-\frac{1}{2}$

$$
|G(m, z)|<a a^{\prime} m^{2} \sum_{\substack{q=1 \\(q, 2)=1}}^{\infty} q^{-3 / 2}<K m^{2} .
$$

Hilfssatz 21. Die Funktion $\Theta(\tau, z)$ ist in der Halbebene $\operatorname{Re} z>-\frac{1}{2}$ analytisch fortsetzbar und dort regulär und eindeutig.

Beweis. Bei festem $\tau$ ist $\Theta(\tau, z)$ in der Halbebene $\operatorname{Re} z>0$ analytisch. Es sei $D$ ein beliebiges beschränktes Gebiet in der Halbebene $\operatorname{Re} z>-\frac{1}{2}$.

Nach den Hilfssätzen 19 und 20 ist das Produkt $F^{r}(m, z) G(m, z)$ in $D$ regulär und dort

$$
|F(m, z) G(m, z)|<K|m|^{2+\varepsilon}, \quad \varepsilon>0 .
$$

Nach (6.2) und Hilfssatz 12 ist

$$
T(m, z)=U\left(z ; 2, \frac{\tau}{4 a a^{\prime}}, m\right)
$$

in $D$ regulär und dort

$$
|T(m, z)|<K e^{-K^{\prime}|m|} .
$$


Aus (6.13) und (6.15) folgt, daß in $D$

$$
\left|T(m, z) \cdot F^{\prime}(m, z) G(m, z)\right|<K|m|^{2+8} e^{-K^{\prime}|m|}, \quad \varepsilon>0 .
$$

Daher konvergiert in $D$ die Reihe

$$
\sum_{\substack{m=-\infty \\ m=0\left(\bmod 4 a a^{\prime}\right)}}^{\infty} T(m, z) F(m, z) G(m, z)
$$

absolut und gleichmäßig; sie stellt dort eine reguläre Funktion von $\approx$ dar. Nach (6.5) ist also

$$
1-2^{-4-2 z}\left(a a^{\prime}\right)^{-2-z} \sum_{\substack{m=-\infty \\ m=0\left(\bmod 4 a a^{\prime}\right)}}^{\infty} T^{\prime}(m, z) F^{\prime}(m, z) G(m, z)
$$

die gesuchte analytische Fortsetzung von $\Theta(\tau, z)$ in die Halbebene $\operatorname{Re} z>-\frac{1}{2}$.

HILfSSATZ 22. Die durch (4.7)-(4.9) definierte Funktion $\Theta(\tau)$ ist für $\operatorname{Im} \tau>0$ regulär und eindeutig.

Beweis. Man wähle in der Halbebene $\operatorname{Re} z>-\frac{1}{2}$ das Gebiet $D$ so, daß es den Punkt $z=0$ enthalte. Nach den Hilfssätzen 18-21, 12 und (6.14) ist

(6.16) $\lim _{z \rightarrow 0} \Theta(\tau, z)=1-2^{-4}\left(a a^{\prime}\right)^{-2} \sum_{\substack{m=-\infty \\ m=0\left(\bmod 4 a a^{\prime}\right)}}^{\infty} T^{\prime}(m, 0) \cdot H^{\prime}(m, 0) G(m, 0)$

$$
=1+\left(2 a a^{\prime}\right)^{-2} \pi^{2} \sum_{\substack{m=1 \\ m \equiv 0\left(\bmod 4 a a^{\prime}\right)}}^{\infty} m e\left(\frac{m \tau}{4 a a^{\prime}}\right) H^{\prime}(m, 0) G^{\prime}(m, 0) .
$$

Aus (6.3), (6.4) und Hilfssatz 13 ergibt sich

$$
F(m, 0) G(m, 0)=\sum_{q=1}^{\infty} A_{q} .
$$

Aus (4.7)-(4.9) und (6.17), (6.18) bekommt man

$$
\Theta(\tau)=\lim _{z \rightarrow \infty} \Theta(\tau, z) .
$$

Die Behauptung folgt jetzt aus (6.19), (6.17) und (6.13).

Hufssatz 23. Für jede Substitution der Gruppe $\Gamma\left(4 a a^{\prime}\right)$ ist

$$
\Theta\left(\frac{\alpha \tau+\beta}{\gamma \tau+\delta}\right)=(\gamma \tau+\delta)^{2} \Theta(\tau) \text {. }
$$

Beweis. Ersetzt man in (6.1)

$$
\tau \quad \text { durch } \frac{a \tau+\beta}{\gamma \tau+\delta}
$$

und schreibt

$$
\text { (6.20) } \quad\left\{\begin{array} { l } 
{ \alpha q + \gamma H = q _ { 0 } , } \\
{ \beta q + \delta H = H _ { 0 } , }
\end{array} \quad \text { d. h. } \quad \left\{\begin{array}{r}
\delta q_{0}-\gamma H_{0}=q, \\
-\beta q_{0}+\alpha H_{0}=H,
\end{array}\right.\right.
$$

so ergibt sich

$$
\text { (6.21) } \Theta\left(\frac{\alpha \tau+\beta}{\gamma \tau+\delta}, z\right)=1-\frac{1}{8 a a^{\prime}} \sum_{\substack{q, H=-\infty \\(H, q)=1}}^{\infty}\left(\frac{\tilde{S}(-H \operatorname{sgn} q,|q|)}{q \frac{q_{0} \tau+H_{0}}{\gamma \tau+\delta}}\right)^{2}\left|\frac{\gamma \tau+\delta}{q_{0} \tau+H_{0}}\right|^{*} .
$$

In (6.21) man kann jetzt $q_{0}$ und $H_{0}$ als neue Summationsbuchstaben statt $q$ und $H$ einführen. Dabei beachte man, daß die Glieder mit $q=0$, $H= \pm 1$ in der Summe auszuschließen sind und betrachte die Fälle $\gamma \neq 0, \gamma=0$ gesondert.

1) Es sei $\gamma \neq 0$. Dann sind in den neuen Summationsbuchstaben die Glieder mit $q_{0}=\gamma, H_{0}=\delta$ und $q_{0}=-\gamma, H_{0}=-\delta$ auszuschließen Die Glieder mit $q=-\gamma, H=\alpha$ und $q=\gamma, H=-\alpha$ erhalten wir jetzt bei $q_{0}=0, H_{0}= \pm 1$. Für $\operatorname{Re} z>0$ ist also

$$
\begin{aligned}
& \Theta\left(\frac{\alpha \tau+\beta}{\gamma \tau+\delta}, z\right)=1-\frac{1}{4 a a^{\prime}}(\gamma \tau+\delta)^{2}|\gamma \tau+\delta|^{z}\left(\frac{\tilde{S}(\alpha \operatorname{sgn} \gamma,|\gamma|)}{\gamma}\right)^{2}- \\
& -\frac{1}{8 a a^{\prime}}(\gamma \tau+\delta)^{2}|\gamma \tau+\delta|^{z} \sum_{\substack{a_{0}, H_{0}=-\infty \\
\left(B_{0}, q_{0}\right)=1}}^{\infty \prime \prime}\left(\frac{\tilde{S}(-H \operatorname{sgn} q,|q|)}{q\left(q_{0} \tau+H_{0}\right)}\right)^{2}\left|q_{0} \tau+H_{0}\right|^{-z},
\end{aligned}
$$

wo zur Abkürzung $H=\alpha H_{0}-\beta q_{0}$ und $q=\delta q_{0}-\gamma H_{0}$ gesetzt ist. Ferner bedeuten die zwei Striche, daß die Glieder mit $q_{0}=0, H_{0}= \pm 1$, mit $q_{0}=\gamma, \mathbf{H}_{0}=\delta$ und mit $q_{0}=-\gamma, H_{0}=-\delta$ auszuschließen sind.

Nach den Hilfssätzen 2 und 3 ist

$$
\text { (6.23) } \quad \tilde{S}^{2}(\alpha \operatorname{sgn} \gamma,|\gamma|)=-4 a a^{\prime} \gamma^{2} \text {. }
$$

Jetzt zeigt man, daß

(6.24) $\quad q_{0}^{2}{\tilde{S^{2}}}^{2}(-H \operatorname{sgn} q,|q|)=q^{2}{\tilde{S^{2}}}^{2}\left(-H_{0} \operatorname{sgn} q_{0},\left|q_{0}\right|\right)$.

In der Tat folgt aus (6.20)

$$
(a, q) \mid q_{0}, \text { d. h. }(a, q)\left|\left(a, q_{0}\right) ; \quad\left(a, q_{0}\right)\right| q \text {, d. h. }\left(a, q_{0}\right) \mid(a, q) ;
$$

$$
(a, q)=\left(a, q_{0}\right) ;
$$


analog hat man

(6.26)

$$
\left(a^{\prime}, q\right)=\left(a^{\prime}, q_{0}\right) .
$$

Andererseits folgt aus (6.20)

Folglich ist

$$
q \equiv q_{0}\left(\bmod 4 a a^{\prime}\right), \quad H \equiv H_{0}\left(\bmod 4 a a^{\prime}\right) .
$$

$$
\begin{gathered}
\frac{q}{(a, q)} \equiv \frac{q_{0}}{\left(a, q_{0}\right)}(\bmod 4), \quad \frac{q}{\left(a^{\prime}, q\right)} \equiv \frac{q_{0}}{\left(a^{\prime}, q_{0}\right)}(\bmod 4) \\
\frac{a^{\prime} H}{\left(a^{\prime}, q\right)} \equiv \frac{a^{\prime} H_{0}}{\left(a^{\prime}, q_{0}\right)}(\bmod 4)
\end{gathered}
$$

und daher

$$
\frac{q^{2}}{(a, q)\left(a^{\prime}, q\right)} \equiv \frac{q_{0}^{2}}{\left(a, q_{0}\right)\left(a^{\prime}, q_{0}\right)}(\bmod 4)
$$$$
\text { (6.28) } \quad \frac{q}{(a, q)}-\frac{a^{\prime} H}{\left(a^{\prime}, q\right)} \equiv \frac{q_{0}}{\left(a, q_{0}\right)}-\frac{a^{\prime} H_{0}}{\left(a^{\prime}, q_{0}\right)}(\bmod 4) \text {. }
$$

Nach (4.11) ist

$\tilde{S}^{2}(-H \operatorname{sgn} q,|q|)$

$$
=\left\{\begin{array}{cl}
q^{2}(a, q)\left(a^{\prime}, q\right)\left(\frac{-1}{|q| /(a, q)}\right)\left(\frac{-1}{|q|\left(a^{\prime}, q\right)}\right) & \text { für } 2 \times q, \\
0 \quad \text { für } 2 \| q \text { oder } & \text { für } 2^{r}\left\|q, 2^{r-1}\right\| a(r>1), \\
2 q^{2}(a, q)\left(a^{\prime}, q\right) i^{\left(\frac{a}{(a, q)}-\frac{a^{\prime} H}{\left(a^{\prime}, q\right)}\right) \operatorname{sgn} q-1} & \text { für } 2^{r}\left\|q, 2^{r}\right\| a(r>1), \\
4 q^{2}(a, q)\left(a^{\prime}, q\right) i^{-\left(\frac{a}{(a, q)}+\frac{a^{\prime}}{\left(a^{\prime}, q\right)}\right) H_{\operatorname{sgn} q}} & \text { für } 2^{r+\boldsymbol{x}}\left\|q, 2^{r-2}\right\| a(r>1),
\end{array}\right.
$$

und

$\tilde{S}^{2}\left(-H_{0} \operatorname{sgn} q_{0},\left|q_{0}\right|\right)$

$$
\left\{\begin{array}{cc}
q_{0}^{2}\left(a, q_{0}\right)\left(a^{\prime}, q_{0}\right)\left(\frac{-1}{\left|q_{0}\right| /\left(a, q_{0}\right)}\right)\left(\frac{-1}{\left|q_{0}\right| /\left(a^{\prime}, q_{0}\right)}\right) & \text { für } 2 \times q_{0}, \\
0 & \text { für } 2 \| q_{0} \text { oder für } 2^{r}\left\|q_{0}, 2^{r-1}\right\| a(r>1), \\
2 q_{0}^{2}\left(a, q_{0}\right)\left(a^{\prime}, q_{0}\right) i^{\left.\left(\frac{q_{0}}{\left(a, q_{0}\right)}-\frac{a^{\prime} B_{0}}{\left(a^{\prime}, q_{0}\right)}\right)\right)_{g g n} q_{0}-1} & \text { für } 2^{r}\left\|q_{0}, 2^{r}\right\| a \quad(r>1), \\
4 q_{0}^{2}\left(a, q_{0}\right)\left(a^{\prime}, q_{0}\right) i^{-\left(\frac{a}{\left(a, q_{0}\right)}+\frac{a^{\prime}}{\left(a^{\prime}, q_{0}\right)}\right) H_{0} \operatorname{sgn} q_{0}} & \text { für } 2^{r+x}\left\|q_{0}, 2^{r-2}\right\| a \quad(r>1) .
\end{array}\right.
$$
(6.24)

Aus diesen Beziehungen und aus (6.25)-(6.28), (6.20) ergibt sich Aus (6.22)-(6.24) folgt

(6.29) $\Theta\left(\frac{\alpha \tau+\beta}{\gamma \tau+\delta}, z\right)=1+(\gamma \tau+\delta)^{2}|\gamma \tau+\delta|^{z}+\frac{1}{4 a a^{\prime}}\left(\gamma^{-1} \tilde{S}(-\delta \operatorname{sgn} \gamma,|\gamma|)\right)^{2}-$

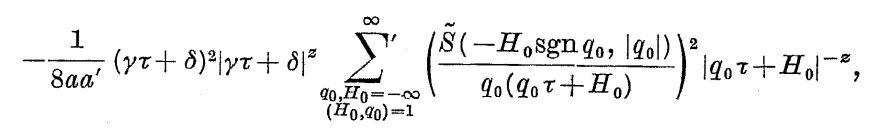

wo der Strich bedeutet, daß nur die Glieder mit $q_{0}=0$ auszuschließen sind.

Schreibt man $q, H$ statt $q_{0}, H_{0}$ und beachtet, daß

$$
\tilde{S^{2}}(-\delta \operatorname{sgn} \gamma,|\gamma|)=-4 a a^{\prime} \gamma^{2},
$$

so bekommt man nach (6.29) und (6.1) für $\operatorname{Re} z>0$

$$
\Theta\left(\frac{\alpha \tau+\beta}{\gamma \tau+\delta}, z\right)=(\gamma \tau+\delta)^{2}|\gamma \tau+\delta|^{z} \Theta(\tau, z) .
$$

Durch Übergang zum Grenzwert $z \rightarrow 0$ ergibt sich hieraus nach (6.19) die. Behauptung.

2) Es sei $\gamma=0$. Wegen $a \equiv \delta \equiv 1\left(\bmod 4 a a^{\prime}\right)$ ist $\alpha=\delta=1$. Man hat daher in den neuen Summationsbuchstaben die Glieder mit $q_{0}=0$, $H_{0}= \pm 1$ wegzulassen, was durch $\Sigma^{\prime}$ angedeutet wird. Es ergibt sich für $\operatorname{Re} z>0$

(6.30) $\Theta(\tau+\beta, z)=1-\frac{1}{8 a a^{\prime}} \sum_{\substack{q_{0}, H_{0}=-\infty \\\left(H_{0}, q_{0}\right)=1}}^{\infty}\left(\frac{\tilde{S}\left(\left(\beta q_{0}-H_{0}\right) \operatorname{sgn} q_{0},\left|q_{0}\right|\right)}{q_{0}\left|q_{0} \tau+H_{0}\right|}\right)^{2}\left|q_{0} \tau+H_{0}\right|^{-z}$.

Wegen

$$
S\left(\left(\beta q_{0}-H_{0}\right) \operatorname{sgn} q_{0},\left|q_{0}\right|\right)=S\left(-H_{0} \operatorname{sgn} q_{0},\left|q_{0}\right|\right)
$$

ist nach (6.30) und (6.1) für $\operatorname{Re} z>0$

$$
\Theta(\tau+\beta, z)=\Theta(\tau, z),
$$

woraus die Behauptung durch Grenzübergang folgt.

Hilfssatz 24. Die Funktion $\Theta(\tau)$ erfüllt die Bedingung 3 von Definition 1. 
Beweis. Folgt aus (4.7) und Hilfssatz 13.

HUFSSATZ 25. Es sei $\alpha \delta-\beta \gamma=1$. Dann gilt in der Umgebung von

$$
\tau=-\frac{\delta}{\gamma} \quad(\gamma \neq 0,(\gamma, \delta)=1)
$$

die Entwicklung

$$
(\gamma \tau+\delta)^{2} \Theta(\tau)=\sum_{n=0}^{\infty} A_{n} e\left(\frac{n}{4 a a^{\prime}} \frac{\alpha \tau+\beta}{\gamma \tau+\delta}\right) .
$$

Beweis. Es sei

$$
L=\left(\begin{array}{ll}
\alpha & \beta \\
\gamma & \delta
\end{array}\right)
$$

eine beliebige Matrix der Gruppe $\Gamma$. Nach Hecke schreibt man

$$
(\gamma \tau+\delta)^{-2} \Theta\left(\frac{\alpha \tau+\beta}{\gamma \tau+\delta}\right)=\Theta \mid L .
$$

Aus dieser Definition ergibt sich für beliebige Matrizen $L_{1}$ und $L_{2}$ von $I$

$$
\Theta\left|L_{1} L_{2}=\left(\Theta \mid L_{1}\right)\right| L_{2} .
$$

Für eine beliebige Matrix $\nabla$ der Gruppe $\Gamma\left(4 a a^{\prime}\right)$ ist nach Hilfssatz 23 (6.32)

$$
\Theta \mid V=\Theta \text {. }
$$

Es sei $U=\left(\begin{array}{ll}1 & 1 \\ 0 & 1\end{array}\right)$. Dann ist $U^{4 a a^{\prime}}=\left(\begin{array}{cc}1 & 4 a a^{\prime} \\ 0 & 1\end{array}\right)$ eine Matrix der Grup-

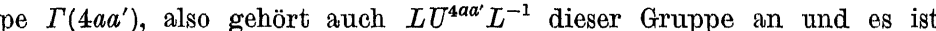
nach $(6.32)$

(6.33)

$$
\Theta \mid L U^{4 a \alpha^{\prime}} L^{-1}=\Theta
$$

Setzt man

so ist nach (6.31)

$$
\Theta \mid L=\varphi(\tau)
$$

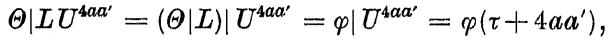

andererseits hat man nach (6.31) und (6.33)

$$
\Theta\left|L U^{4 a a^{\prime}}=\Theta\right| L=\varphi(\tau) \text {. }
$$

Die reguläre Funktion $\varphi(\tau)$ hat mithin die Periode $4 a a^{\prime}$. Daher ist sie in eine absolut konvergente Fourierreihe entwickelbar:

$(6.34)$

$$
(\gamma \tau+\delta)^{-2} \Theta\left(\frac{\alpha \tau+\beta}{\gamma \tau+\delta}\right)=\sum_{n=-\infty}^{\infty} A_{n} e\left(\frac{\tau n}{4 a a^{\prime}}\right) .
$$

Hierbei sind die Koeffizienten $A_{n}$ durch das Integral

$$
A_{n}=\int_{\tau_{0}-2 a a^{\prime}}^{\tau_{0}+2 a a^{\prime}}(\gamma \tau+\delta)^{-2} \Theta\left(\frac{\alpha \tau+\beta}{\gamma \tau+\delta}\right) e\left(-\frac{\tau n}{4 a a^{\prime}}\right) d \tau
$$

gegeben, wo $\tau_{0}$ ein beliebiger Punkt der oberen Halbebene ist.

Setzt man $\tau=\mu_{1}+i \mu_{2}\left(\mu_{2}>0\right)$, so ist nach (6.19), (6.17) und (6.13)

$$
|\Theta(\tau)| \leqslant 1+K \sum_{m=1}^{\infty} m^{4} \exp \left(-\frac{2 \pi m \mu_{2}}{4 a a^{\prime}}\right) .
$$

Wegen

ist folglich

$$
\exp \left(\frac{2 \pi m \mu_{2}}{4 a a^{\prime}}\right) \geqslant K m^{6} \mu_{2}^{6}
$$

$$
|\Theta(\tau)| \leqslant 1+K \sum_{m=1}^{\infty} m^{-2} \mu_{2}^{-6}<1+K \mu_{2}^{-6} .
$$

Es sei $\tau_{0}=-\delta / \gamma+i \eta_{0}\left(\eta_{0}>0\right)$. In (6.35) ist dann

folglich

$$
\tau=\xi+i \eta_{0} ; \quad-2 a a^{\prime}-\delta / \gamma \leqslant \xi \leqslant 2 a a^{\prime}-\delta / \gamma,
$$

d. h.

$$
\gamma \tau+\delta=(\gamma \xi+\delta)+i \gamma \eta_{0} ; \quad-2 a a^{\prime} \gamma \leqslant \gamma \xi+\delta \leqslant 2 a a^{\prime} \gamma,
$$

In (6.35) ist also

$$
\begin{aligned}
& \operatorname{Im}\left(-\frac{1}{\gamma \tau+\delta}\right)=\frac{\gamma \eta_{0}}{(\gamma \xi+\delta)^{2}+\gamma^{2} \eta_{0}^{2}} \geqslant \frac{\eta_{0}}{\gamma\left(4 a^{2} a^{\prime 2}+\eta_{0}^{2}\right)} \quad \text { für } \quad \gamma>0, \\
& \operatorname{Im}\left(\frac{1}{\gamma \tau+\delta}\right)=\frac{-\gamma \eta_{0}}{(\gamma \xi+\delta)^{2}+\gamma^{2} \eta_{0}^{2}} \geqslant \frac{\eta_{0}}{-\gamma\left(4 a^{2} a^{\prime 2}+\eta_{0}^{2}\right)} \quad \text { für } \quad \gamma<0,
\end{aligned}
$$

d. h.

$\operatorname{Im}\left(\frac{\alpha \tau+\beta}{\gamma \tau+\delta}\right)=\operatorname{Im}\left(\frac{\alpha}{\gamma}-\frac{1}{\gamma(\gamma \tau+\delta)}\right)=\operatorname{Im}\left(-\frac{1}{\gamma(\gamma \tau+\delta)}\right) \geqslant \frac{\eta_{0}}{\gamma^{2}\left(4 a^{2} a^{\prime 2}+\eta_{0}^{2}\right)}$.

Nach (6.36) ist somit

Ferner ist

(6.38)

$$
\left|\Theta\left(\frac{\alpha \tau+\beta}{\gamma \tau+\delta}\right)\right| \leqslant 1+K\left(\eta_{0}^{-1} \gamma^{2}\left(4 a^{2} a^{\prime 2}+\eta_{0}^{2}\right)\right)^{6} .
$$

$$
|\gamma \tau+\delta|^{2} \geqslant \gamma^{2} \eta_{0}^{2}
$$

Acta Arithmetica $\mathrm{V}$ 
Aus (6.37) und (6.38) folgt, daß in (6.35)

$$
\left|(\gamma \tau+\delta)^{-2} \Theta\left(\frac{\alpha \tau+\beta}{\gamma \tau+\delta}\right)\right| \leqslant \eta_{0}^{-2}+K \eta_{0}^{-8}\left(4 a^{2} a^{\prime 2}+\eta_{0}^{2}\right)^{6} ;
$$

folglich

$$
\left|A_{n}\right| \leqslant \exp \left(\frac{\pi \eta_{0} n}{2 a a^{\prime}}\right)\left(\eta_{0}^{-2}+K \eta_{0}^{-8}\left(4 \cdot a^{2} a^{\prime 2}+\eta_{0}^{2}\right)^{6}\right) 4 a a^{\prime},
$$

d. h. $A_{n} \rightarrow 0$, wenn $\eta_{0} \rightarrow \infty$ und $n<0$.

Man hat somit

$$
(\gamma \tau+\delta)^{-2} \Theta\left(\frac{\alpha \tau+\beta}{\gamma \tau+\delta}\right)=\sum_{n=0}^{\infty} A_{n} e\left(\begin{array}{c}
\tau \eta n \\
4 a a^{\prime}
\end{array}\right)
$$

Ersetzt man hier

$$
\alpha, \delta, \beta, \gamma, \tau \quad \text { durch } \quad \delta, \alpha,-\beta,-\gamma, \frac{\alpha \tau+\beta}{\gamma \tau+\delta},
$$

so ergibt sich die Behauptung.

HILFSSATZ 26. Die Frunktion $\Theta(\tau)$ ist eine ganze Modulform der stufe $4 a a^{\prime}$ und der Dimension -2.

Beweis. Die Behauptung folgt aus den Irilfssätzon $22,23,24,25$ und Definition 1.

In den folgenden Paragraphen bezeichnen $\alpha, \beta, \gamma, l$, mit $\Lambda$ usnahmo von Hilfssatz 31 , nichtnegative ganze Zahlen; $n$ natürliche Zahlen; $a^{\prime}, w$ positive ungerade Zahlen.

§7. In diesem Paragraphen wird mittels der Kloostermanschen Methode die durch (4.8)-(4.9) definierte singuläre Reihe $\varrho(M)$ summiert.

HIIFSSATZ 27. Es sei

$$
\chi_{p}=1+A_{p}+A_{p^{2}}+\ldots
$$

Dann ist

$$
\sum_{q=1}^{\infty} A_{q}=\prod_{p} x_{p}
$$

Beweis. Für $\left(q, q^{\prime}\right)=1$ folgt aus $(4.9)$

$$
A_{q} \cdot A_{q^{\prime}}=\left(q q^{\prime}\right)^{-4} \sum_{h \bmod q}^{\prime} \sum_{h^{\prime} \bmod q^{\prime}}^{\tilde{S}^{2}}(h, q) \tilde{\delta^{2}}\left(h^{\prime}, q^{\prime}\right) e\left(-\frac{I T M}{4 a a^{\prime} q q^{\prime}}\right),
$$

wo $H=h q^{\prime}+h^{\prime} q$ ein reduziertes Restsystem mod $q q^{\prime}$ durchläuft. Nach
Hilfssatz 4 ist

$$
A_{q} \cdot A_{q^{\prime}}=\left(q q^{\prime}\right)^{-4} \sum_{H \bmod q q^{\prime}}^{\prime} \tilde{S}^{2}\left(H, q q^{\prime}\right) e\left(-\frac{H M}{4 a a^{\prime} q q^{\prime}}\right)=A_{q q^{\prime}} \cdot
$$

Daraus ergibt sich nach Hilfssatz 13 die Behauptung.

HILFSSATz 28. Es sei $M=4 a a^{\prime} n, n=2^{a} m, a=2^{\gamma} b$. Dann ist

$$
\chi_{2}=\left\{\begin{array}{lll}
1+(-1)^{\left(m-a^{\prime}\right) / 2} & \text { für } \quad 0 \leqslant \alpha \leqslant \gamma-2, \\
1 & \text { für } \quad \gamma-2<\alpha<\gamma+1, \\
1+(-1)^{\left(b+a^{\prime}\right) / 2} \cdot 2^{\gamma-\alpha}\left(2^{\alpha-\gamma}-3\right) & \text { für } \quad a \geqslant \gamma+1 .
\end{array}\right.
$$

Beweis. Aus (7.1), (4.9) und (6.3) folgt

$$
\chi_{2}=F^{\prime}(M, 0) \text {. }
$$

Aus (6.11) und (1.3) ergibt sich also

$$
\begin{aligned}
\chi_{2}=1 & +\sum_{\lambda=2}^{\gamma} 2^{1-\lambda} \sum_{h \operatorname{mod2}}^{\lambda} e\left(\frac{h b}{4}-\frac{h M}{2^{\lambda+\gamma+2}}\right)+ \\
& +\sum_{\lambda=\gamma+2}^{\infty} 2^{\gamma+2-2 \lambda}(-1)^{\left(b+a^{\prime}\right) / 2} c\left(\frac{M}{2^{\gamma+2}}, 2^{\lambda}\right) .
\end{aligned}
$$

Hier ist

$$
\begin{aligned}
2^{1-\lambda} \sum_{h \bmod 2^{\lambda}}^{\prime} e\left(\frac{h b}{4}-\frac{h M}{2^{\lambda+\gamma+2}}\right) & =2^{1-\lambda} e\left(\frac{b}{4}-\frac{M}{2^{\lambda+\gamma+2}}\right)^{2^{\lambda-1}-1} \sum_{x=0} e\left(\frac{x b}{2}-\frac{x M}{2^{\lambda+\gamma+1}}\right) \\
& =\left\{\begin{array}{cll}
(-1)^{\left(m-a^{\prime}\right) y^{2}} & \text { für } & \lambda=\alpha+2, \\
0 & \text { für } & \lambda \neq \alpha+2
\end{array}\right.
\end{aligned}
$$

und nach Hilfssatz 7

$$
2^{\gamma+2-2 \lambda}(-1)^{\left(b+a^{\prime}\right) / 2} c\left(\frac{M}{2^{\gamma+2}}, 2^{\lambda}\right)=\left\{\begin{array}{cll}
0 & \text { für } & \alpha<\lambda-1, \\
(-1)^{\left(b+a^{\prime}\right) / 2+1} \cdot 2^{-\lambda+\gamma+1} & \text { für } & \alpha=\lambda-1, \\
(-1)^{\left(b+a^{\prime}\right) / 2} \cdot 2^{-\lambda+\gamma+1} & \text { für } & \alpha>\lambda-1 .
\end{array}\right.
$$

Die zwei ersten Behauptungen ergeben sich aus (7.2)-(7.4). Ist $\alpha \geqslant \gamma+1$, so folgt aus (7.2)-(7.4)

$$
\begin{aligned}
\chi_{2} & =1+\sum_{\lambda=\gamma+2}^{a}(-1)^{\left(b+a^{\prime}\right) / 2} \cdot 2^{-\lambda+\gamma+1}+(-1)^{\left(b+a^{\prime}\right) / 2+1} \cdot 2^{\gamma-\alpha} \\
& =1+(-1)^{\left(b+a^{\prime}\right) / 2} \cdot 2^{\gamma-a}\left(2^{\alpha-\gamma}-3\right),
\end{aligned}
$$

d. h. die dritte Behauptung 
HIIFSSATZ 29. Es sei $\left(a, a^{\prime}\right)=1, p>2, p^{\beta}|| n, p^{l} \| a a^{\prime}$. Dann ist

$$
\chi_{p}=\left\{\begin{array}{cl}
\left(1-p^{-1}\right)(\beta+1) & \text { für } \quad l \geqslant \beta+1, p \equiv 1(\bmod 4), \\
1+p^{-1} & \text { für } \quad l \geqslant \beta+1, p \equiv 3(\bmod 4), 2 \mid \beta, \\
0 & \text { für } \quad l \geqslant \beta+1, p \equiv 3(\bmod 4), 2+\beta,
\end{array}\right.
$$

$$
\chi_{p}=\left\{\begin{array}{lll}
\left(1-p^{-1}\right)\left\{l+\left(1+p^{-1}\right)\left(1+p^{-1}+\right.\right. & & \\
\left.\left.+p^{-2}+\ldots+p^{l-\beta}\right)\right\} & \text { für } & l \leqslant \beta, p=1(\bmod 4), \\
\left(1+p^{-1}\right)\left(1-p^{l-\beta-1}\right) & \text { für } & l \leqslant \beta, p=3(\bmod 4), 2 \mid l, \\
p^{l-\beta-1}\left(1+p^{-1}\right) & \text { für } & l \leqslant \beta, p=3(\bmod 4), 2+l .
\end{array}\right.
$$

Beweis. Aus (7.1), (4.9), (6.4), (6.12) und Filfssatz 7 ergibt sioh

$$
\begin{aligned}
\chi_{p}=1+ & \sum_{\lambda=1}^{\beta}\left(\frac{-1}{p^{2 \lambda} /\left(p^{\lambda}, a\right)\left(p^{\lambda}, a^{\prime}\right)}\right)\left(p^{\lambda}, a\right)\left(p^{\lambda}, a^{\prime}\right) p^{-\lambda-1}(p-1)- \\
& -\left(\frac{-1}{p^{2(\beta+1)} /\left(p^{\beta+1}, a\right)\left(p^{\beta+1}, a^{\prime}\right)}\right)\left(p^{\beta+1}, a\right)\left(p^{\beta+1}, a^{\prime}\right) p^{-\beta-2} .
\end{aligned}
$$

Wegen $\left(a, a^{\prime}\right)=1$ folgt aus (7.5) für $l \geqslant \beta+1$

$$
\chi_{p}=1+\sum_{\lambda=1}^{\beta}\left(\frac{-1}{p}\right)^{\lambda}\left(1-p^{-1}\right)-\left(\frac{-1}{p}\right)^{\beta+1} p^{\cdots-1},
$$

woraus sich für $l \geqslant \beta+1$ die Behauptung ergibt.

Für $l \leqslant \beta$ folgt aus $(7.5)$

$$
\chi_{p}=1+\sum_{\lambda=1}^{l}\left(\frac{-1}{p}\right)^{\lambda}\left(1-p^{-1}\right)+\sum_{\lambda=l+1}^{\beta}\left(\frac{-1}{p}\right)^{l} p^{l-\lambda-1}(p-1)-\left(\frac{-1}{p}\right)^{l} p^{l-\beta-2} .
$$

Es ist somit: für $p \equiv 1(\bmod 4)$

$$
\begin{aligned}
\chi_{p} & =1+\left(1-p^{-1}\right) l+\left(p^{-1}+p^{-2}+\ldots+p^{l-\beta}\right)-p^{-2}\left(1+p^{-1}+p^{-2}+\ldots+p^{l \cdots \beta}\right) \\
& =\left(1-p^{-1}\right) l+\left(1-p^{-2}\right)\left(1+p^{-1}+p^{-2}+\ldots+p^{l \cdots \beta}\right)
\end{aligned}
$$

für $p \equiv 3(\bmod 4), 2 \mid l$

$$
\begin{aligned}
\chi_{p} & =1+(p-1)\left(p^{-2}+p^{-3}+\ldots+p^{l-\beta-1}\right)-p^{l-\beta \cdots 2} \\
& =\left(1-p^{-2}\right)\left(1+p^{-1}+p^{-2}+\cdots+p^{l-\beta}\right)=\left(1+p^{-1}\right)\left(1-p^{l \cdots \beta-1}\right) ;
\end{aligned}
$$

für $p \equiv 3(\bmod 4), 2 \times l$

$$
\chi_{p}=p^{-1}-(p-1)\left(p^{-2}+p^{-3}+\ldots+p^{l-\beta-1}\right)+p^{l-\beta-2}=p^{l \cdots \beta-1}+p^{l-\beta \cdots 2} .
$$

Hilfissatz 30. Es sei $M=4 a a^{\prime} n, n=2^{a} m=2^{a} v u, a=2^{\gamma} b$,

$$
v=\prod_{\substack{p|n, p| b a^{\prime} \\ p>2}} p^{\beta}, \quad u=\prod_{p \mid n, p \times 2 b a^{\prime}} p^{\beta}
$$

Dann ist

$$
\varrho(M)=\frac{2^{a+3}}{a a^{\prime}} v \sigma(u) \chi_{2} \prod_{p \mid b a^{\prime}} \chi_{p} \prod_{p \mid b a^{\prime}}\left(1-p^{-2}\right)^{-1},
$$

wobei die Werte von $\chi_{2}$ und $\chi_{p}$ in den Hilfssätzen 28 und 29 angegeben werden.

Beweis. Ist $p^{\beta} \| n, p \nmid 2 b a^{\prime}$, so folgt aus (7.5)

$$
\chi_{p}=1+\sum_{\lambda=1}^{\beta} p^{-\lambda-1}(p-1)-p^{-\beta-2}=\left(1-p^{-2}\right) \sum_{d \mid p^{\beta}} d^{-1} .
$$

Für $p \times 2 b a^{\prime} n$ ist daher

$$
\chi_{p}=1-p^{-2}
$$

Aus Hilfssatz 27, (7.7) und (7.8) ergibt sich

$$
\begin{aligned}
\sum_{q=1}^{\infty} A_{q} & =\chi_{2} \prod_{p \mid b a^{\prime}} \chi_{p} \prod_{p \times 2 b a^{\prime}} \chi_{p} \\
& =\chi_{2} \prod_{p \mid b a^{\prime}} \chi_{p} \prod_{p>2}\left(1-p^{-2}\right) \prod_{p \mid b a^{\prime}}\left(1-p^{-2}\right)^{-1} \sum_{d \mid u} d^{-1} \\
& =8 \pi^{-2} \chi_{2} \prod_{p \mid b a^{\prime}} \chi_{p} \prod_{p \mid b a^{\prime}}\left(1-p^{-2}\right)^{-1} \sum_{d \mid u} d^{-1} .
\end{aligned}
$$

Aus (4.8), (7.9) und (1.1) folgt die Behauptung.

§ 8. In diesem Paragraphen wird die Darstellung der Zahlen durch die Form

untersiucht.

$$
x_{1}^{2}+x_{2}^{2}+5 x_{3}^{2}+5 x_{4}^{2}
$$

HulfSSATZ 31. Die Frunktion

(8.1) $\quad \psi(\tau ; 1,5)=\vartheta_{00}^{2}(\tau ; 0,2) \vartheta_{00}^{2}(\tau ; 0,10)-\Theta(\tau ; 5)-$

$$
-A \vartheta_{21}^{2}(\tau ; 0,10) \vartheta_{61}^{2}(\tau ; 0,10)
$$

ist bei konstantem $A$ eine ganze Modulform der Stufe 20 und der Dimension -2 .

Beweis. Nach den Hilfssätzen 17 und 26 sind die Funktionen $\vartheta_{00}^{2}(\tau ; 0,2) \vartheta_{00}^{2}(\tau ; 0,10)$ und $\Theta(\tau ; 5)$ ganze Modulformen der Stufe 20 und der Dimension -2. 
Wegen (1.5) erfüllt die Funktion $\vartheta_{21}^{2}(\tau ; 0,10) \vartheta_{61}^{2}(\tau ; 0,1.0)$ die Bedingungen 1 und 3 von Definition 1.

Nach den Hilfssätzen 15 und 3 gilt für jede Substitution der Gruppe $\Gamma(20)$

$$
(\gamma \tau+\delta)^{-2} \vartheta_{21}^{2}\left(\frac{\alpha \tau+\beta}{\gamma \tau+\delta} ; 0,10\right) \vartheta_{61}^{2}\left(\frac{\alpha \tau+\beta}{\gamma \tau+\delta} ; 0,10\right)=\vartheta_{21}^{2}(\tau ; 0,10) \vartheta_{61}^{2}(\tau ; 0,10) .
$$

Die Bedingung 2 von Definition 1 ist also erfüllt.

Nach Hilfssatz 16 gilt in der Umgebung von

$$
\tau=-\frac{\delta}{\gamma} \quad(\gamma \neq 0,(\gamma, \delta)=1, \alpha \delta-\beta \gamma=1)
$$

die Entwicklung

$$
(\gamma \tau+\delta)^{2} \vartheta_{21}^{2}(\tau ; 0,10) \vartheta_{61}^{2}(\tau ; 0,10)=\sum_{n=0}^{\infty} C_{n} e\left(\frac{n}{20} \cdot \frac{\alpha \tau+\beta}{\gamma \tau+\delta}\right) .
$$

Die Bedingung 4 von Definition 1 ist mithin ebenfalls erfüllt.

Die Funktion $\vartheta_{21}^{2}(\tau ; 0,10) \vartheta_{61}^{2}(\tau ; 0,10)$ ist daher eine ganze Modulform der Stufe 20 und der Dimension -2 .

\section{SATZ 1.}

(8.2) $\quad \vartheta_{00}^{2}(\tau ; 0,2) \vartheta_{00}^{2}(\tau ; 0,10)=\Theta(\tau ; 5)+\frac{8}{3} \vartheta_{21}^{2}(\tau ; 0,10) \vartheta_{61}^{2}(\tau ; 0,1.10)$.

Beweis. Nach den Hilfssätzen 31 und 1 ist die Funktion $\psi(\tau ; 1,5)$ identisch Null, wenn sie in ihrem Fundamentalbereich mohr als

$$
\frac{2}{24} 20^{3} \prod_{p \mid 20}\left(1-p^{-2}\right)=480
$$

Nullstellen hat. Dazu reicht es hin, daß $\tau=i \infty$ ein mehr als 480 -facher Nullpunkt sei. Es soll gezeigt werden, daß bei geeigneter Wahl von. $A$ in der Entwickling von $\psi(\tau ; 1,5)$ nach Potenzen von $Q$ die Glieder mit $Q^{M}$, $M \leqslant 480$ verschwinden.

Setzt man in den Hilfssätzen 28,29 und 30

$$
a=1, \quad a^{\prime}=5, \quad \gamma=0, \quad b=1, \quad v=5^{\beta}, \quad l=1,
$$

d. h. '

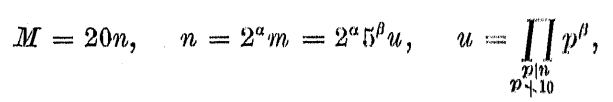

so ergibt sich

$$
\varrho(M)=\frac{2^{a}}{3} 5^{\beta+1} \sigma(u) \chi_{2} \chi_{5} .
$$

Hierbei ist

$$
\chi_{2}= \begin{cases}1 & \text { für } \quad a=0 \\ 3 \cdot 2^{-a} & \text { für } \quad a>0\end{cases}
$$

und

$$
\chi_{5}=\left\{\begin{array}{l}
\frac{4}{5} \quad \text { für } \quad \beta=0, \\
\frac{4}{5}\left\{1+\frac{6}{5}\left(1+5^{-1}+\ldots+5^{-\beta+1}\right)\right\}=2\left(1-3 \cdot 5^{-\beta-1}\right) \text { für } \beta>0,
\end{array}\right.
$$

also in beiden Fällen

$$
\chi_{5}=2\left(1-3 \cdot 5^{-\beta-1}\right) .
$$

Aus (8.3)-(8.5) folgt

$$
\varrho(M)=\left\{\begin{array}{lll}
\frac{2}{3}\left(5^{\beta+1}-3\right) \sigma(u) & \text { für } & \alpha=0 \\
2\left(5^{\beta+1}-3\right) \sigma(u) & \text { für } & \alpha>0 .
\end{array}\right.
$$

Setzt $\operatorname{man} Q=e(\tau / 20)$, so ergibt sich aus (1.4)

(8.7) $\vartheta_{00}^{2}(\tau ; 0,2) \vartheta_{00}^{2}(\tau ; 0,10)=\left(\sum_{k=-\infty}^{\infty} Q^{20 k^{2}}\right)^{2}\left(\sum_{k=-\infty}^{\infty} Q^{100 k^{2}}\right)^{2}$

$$
\begin{aligned}
= & 1+4 Q^{20}+4 Q^{40}+4 Q^{80}+12 Q^{100}+16 Q^{120}+ \\
& +16 Q^{140}+4 Q^{160}+20 Q^{180}+44 Q^{200}+16 Q^{220}+ \\
& +16 Q^{240}+24 Q^{260}+32 Q^{280}+64 Q^{300}+4 Q^{320}+ \\
& +8 Q^{340}+52 Q^{360}+16 Q^{380}+44 Q^{400}+32 Q^{420}+ \\
& +48 Q^{440}+48 Q^{460}+16 Q^{480}+\ldots, \\
= & \left(\sum_{k=-\infty}^{\infty}(-1)^{k} Q^{(10 k+1)^{2}}\right)^{2}\left(\sum_{k=-\infty}^{\infty}(-1)^{k} Q^{(10 k+3)^{2}}\right)^{2} \\
= & Q^{20}-2 Q^{60}-Q^{100}+2 Q^{140}+Q^{180}+2 Q^{260}+ \\
& +2 Q^{300}-6 Q^{340}-4 Q^{380}-4 Q^{420}+6 Q^{460}+ \\
& +Q^{500}+\ldots
\end{aligned}
$$

(8.8) $\vartheta_{21}^{2}(\tau ; 0,10) \vartheta_{61}^{2}(\tau ; 0,10)=\left(\sum_{k=-\infty}^{\infty}(-1)^{k} Q^{(10 k+1)^{2}}\right)^{2}\left(\sum_{k=-\infty}^{\infty}(-1)^{k} Q^{(10 k+3)^{2}}\right)^{2}$

Berechnet man die Werte von $\varrho(M)$ mittels der Formeln (8.6) und setzt sie in (4.7) ein, so bekommt man

(8.10) $\Theta(\tau ; 5)=1+\frac{4}{3} Q^{20}+4 Q^{40}+\frac{16}{3} Q^{60}+4 Q^{80}+\frac{44}{3} Q^{100}+16 Q^{120}+\frac{32}{3} Q^{140}+$

$$
\begin{aligned}
& +4 Q^{160}+\frac{52}{3} Q^{180}+44 Q^{200}+16 Q^{220}+16 Q^{240}+\frac{56}{3} Q^{260}+ \\
& +32 Q^{280}+\frac{176}{3} Q^{300}+4 Q^{320}+24 Q^{340}+52 Q^{360}+\frac{80}{3} Q^{380}+ \\
& +44 Q^{400}+\frac{128}{3} Q^{420}+48 Q^{440}+32 Q^{460}+16 Q^{480}+\ldots
\end{aligned}
$$


Man wähle jetzt die Konstante $A$ so, daß der Koeffizienti von $Q^{20}$ in der Entwicklung der Funktion, $\psi(\tau ; 1,5)$ verschwindet. Nach (8.1), (8.7) - (8.10) kommt dies darauf hinaus, dat

$$
4-A-\frac{4}{3}=0 \text {. }
$$

Nimmt man folglich $A=\frac{8}{3}$, so läßt sich ohne schwierigkeit nachprüfen, daß nach (8.1) und (8.7)-(8.10) alle Koeflizienten von $Q^{M}, M$. 5480 , gleich Null sind.

SATZ 1a. Es sei $n=2^{\alpha} 5^{\beta} u,(u, 10)=1, M=20$. Dann ist

$$
r(n)= \begin{cases}\frac{2}{3}\left(5^{\beta+1}-3\right) \sigma(u)+\frac{8}{3} \nu(M) & \text { für } \quad a=0, \\ 2\left(5^{\beta+1}-3\right) \sigma(u) & \text { für } \quad a>0,\end{cases}
$$

wo $v(M)$ den Koeffizienten von $Q^{M}$ in der Entwioklung der Huktion. $\vartheta_{21}^{2}(\tau ; 0,10) \vartheta_{61}^{2}(\tau ; 0,10)$ nach Potenzen von $Q$ bezeichnet.

Bemerkung. In den von Kloosterman gegebenen Formeln für $r(n)$ tritt statt $\nu(M)$ der Koeffizient $\eta_{n}$ von $q^{n}$ in $\frac{1}{5} \vartheta_{1}^{2}\left(\frac{1}{5} \mid \tau\right) \vartheta_{1}^{2}\left(\frac{2}{5} \mid \tau\right)$ auf, wobei $q=Q^{20}$ und $\vartheta_{1}(z \mid \tau)$ eine Jacobische Thetafunktion ist.

Beweis. Aus (8.2), (4.2), (4.3), (4.7) ergibt sich

$$
r(n)=\varrho(M)+\frac{8}{3} \nu(M) .
$$

Aus (8.8) folgt

$\vartheta_{21}^{2}(\tau ; 0,10) \vartheta_{61}^{2}(\tau ; 0,10)=Q^{20}\left(\sum_{k=-\infty}^{\infty}(-1)^{k} Q^{20 k(5 / k+1)}\right)^{2}\left(\sum_{k=1}^{\infty}(-1)^{k} Q^{20 k(5 k+3)}\right)^{2}$

$\vartheta_{21}^{2}(\tau ; 0,10) \vartheta_{61}^{2}(\tau ; 0,10)$ ist also eine ungerade Funktion von ( ${ }^{20}$. Dah. enthält ihre Entwicklung nach Potenzen von $Q$ keine geraden Potionzon von $Q^{20}$, d. h. es ist

$$
v(M)=0 \text { für gerade } n .
$$

Aus (8.12), (8.6) und (8.13) ergibt sich dio Bohauptung.

§9. In diesem Paragraphen wird die Darstellung der Zahlen durah die Form

$$
x_{1}^{2}+x_{2}^{2}+6 x_{3}^{2}+6 x_{i}^{2}
$$

untersucht.

HILFSSATZ 32. Die Funlition

(9.1) $\quad \psi(\tau ; 1,6)=\vartheta_{00}^{2}(\tau ; 0,2) \vartheta_{00}^{2}(\tau ; 0,12)-\Theta(\tau ; 6)$

$$
-A \vartheta_{01}(\tau ; 0,12) \vartheta_{11}^{2}(\tau ; 0,12) \vartheta_{01}(\tau ; 0,12)
$$

ist bei konstantem $A$ eine ganze Modulform der Stufe 24 und der Dimension $-2$

Der Beweis verläuft ähnlich, wie bei Hilfssatz 31 .

SATZ 2.

(9.2) $\quad \vartheta_{00}^{2}(\tau ; 0,2) \vartheta_{00}^{2}(\tau ; 0,12)$

$$
=\Theta(\tau ; 6)+2 \vartheta_{01}(\tau ; 0,12) \vartheta_{41}^{2}(\tau ; 0,12) \vartheta_{81}(\tau ; 0,12) .
$$

Beweis. Nach den Hilfssätzen 32 und 1 ist die Funktion $\psi(\tau ; 1,6)$ identisch Null, wenn sie in ihrem Fundamentalbereich mehr als

$$
\frac{2}{24} 24^{3} \prod_{p \mid 24}\left(1-p^{-2}\right)=768
$$

Nullstellen hat. Dazu reicht es hin, daß $\tau=i \infty$ ein mehr als 768 -facher Nullpunkt sei. Es soll gezeigt werden, daß bei geeigneter Wahl von $A$ in der Entwicklung von $\psi(\tau ; 1,6)$ nach Potenzen von $Q$ die Glieder mit $Q^{M}, M \leqslant 768$ verschwinden.

Setzt man in den Hilfssätzen 28, 29 und 30

$$
a=6, \quad a^{\prime}=1, \quad \gamma=1, \quad b=3, \quad v=3^{\beta}, \quad l=1,
$$

$$
M=24 n, \quad n=2^{a} m=2^{a} 3^{\beta} u, \quad u=\prod_{\substack{p / n \\ p \nmid 6}} p^{\beta},
$$

so ergibt sich

$$
\varrho(M)=2^{a-1} 3^{\beta+1} \sigma(u) \chi_{2} \chi_{3} .
$$

Hierbei ist

$$
\chi_{2}=\left\{\begin{array}{lll}
1 & \text { für } & \alpha=0,1, \\
2^{-a+1}\left(2^{\alpha}-3\right) & \text { für } & \alpha>1
\end{array}\right.
$$

und

$$
\chi_{3}= \begin{cases}\frac{4}{3} & \text { für } \quad \beta=0, \\ 4 \cdot 3^{-\beta-1} & \text { für } \quad \beta>0,\end{cases}
$$

also in beiden Fällen

$$
\chi_{3}=4 \cdot 3^{-\beta-1} .
$$

Aus (9.3)-(9.5) folgt

$$
\varrho(M)=\left\{\begin{array}{lll}
2^{\alpha+1} \sigma(u) & \text { für } & a=0,1 \\
4\left(2^{\alpha}-3\right) \sigma(u) & \text { für } & a>1 .
\end{array}\right.
$$


Setzt man $Q=e(\tau 24)$, so ergibt sich aus (1.4)

$$
\begin{aligned}
& \vartheta_{00}^{2}(\tau ; 0,2) \vartheta_{00}^{2}(\tau ; 0,12)=\left(\sum_{k=-\infty}^{\infty} Q^{24 k^{2}}\right)^{2}\left(\sum_{k=\infty}^{\infty} Q^{14 \cdot k k^{2}}\right)^{2} \\
& =1+4 Q^{24}+4 Q^{48}+4 Q^{96}+8 Q^{120}+4 Q^{144}+1.6 Q^{16 r} \ldots \\
& +20 Q^{192}+4 Q^{216}+24 Q^{240}+32 Q^{204}+4 Q^{288}+ \\
& +24 Q^{312}+32 Q^{336}+16 Q^{360}+52 Q^{344}+40 Q^{304}+ \\
& +4 Q^{432}+32 Q^{456}+24 Q^{480}+16 Q^{504}+48 Q Q^{524}+1 \\
& +32 Q^{552}+20 Q^{576}+60\left(Q^{600}+56\left(Q^{620}+32 Q^{172}+\right.\right. \\
& +72 Q^{690}+24 Q^{720}+80\left(Q^{744}+116()^{704}+\ldots,\right.
\end{aligned}
$$

(9.8) $\quad \vartheta_{01}(\tau ; 0,12) \vartheta_{41}^{2}(\tau ; 0,12) \vartheta_{01}(\tau ; 0,12)$

$$
\begin{aligned}
& =\sum_{k=-\infty}^{\infty}(-1)^{k} Q^{144 k^{2}}\left(\sum_{k=-\infty}^{\infty}(-1)^{k} Q^{(12 k+2)^{2}}\right)^{2} \sum_{k=1}^{\infty}(-\cdots-1)^{k}\left(Q^{(12 k \cdot 1-4)^{2}}\right. \\
& =Q^{24}-Q^{72}-2 Q^{120}+Q^{216}+4 Q^{264}-2 Q^{312}+2 Q^{360}+2 Q^{408}-4 Q^{450} \ldots \\
& -8 Q^{552}-Q^{600}-Q^{648}+6 Q^{696}+8 Q^{744}+2 Q^{702}+\cdots
\end{aligned}
$$

Berechnet man die Werte von. $\varrho(M)$ mittels der firmolu (9.6) und setzt sie in (4.7) ein, so bekommt man

$$
\begin{aligned}
\Theta(\tau ; 6)= & +2 Q^{24}+4 Q^{48}+2 Q^{72}+4 Q^{90}+12\left(Q^{120}+4 Q^{144}+16\left(Q^{164}+\right.\right. \\
& +20 Q^{192}+2 Q^{216}+24 Q^{240}+24 Q^{264}+4 Q^{288}+28\left(Q^{312}+\right. \\
& +32 Q^{336}+12 Q^{360}+52 Q^{384}+36 Q^{408}+4 Q^{432}+40\left(Q^{450}+\right. \\
& +24 Q^{480}+16 Q^{504}+48 Q^{528}+48 Q^{562}+20 Q^{570}+62 Q^{000}+ \\
& +56 Q^{624}+2 Q^{648}+32 Q^{672}+60 Q^{606}+24 Q^{720}+64 Q^{744}+ \\
& +116 Q^{768}+\ldots
\end{aligned}
$$

Man wähle jetzt die Konstante $A$ so, daßs der Kooffizient von $Q^{24}$ in der Entwicklung der Funktion $\psi(\tau ; 1,6)$ verschwindet. Nach (9.1), (9.7)-(9.9) kommt dies darauf hinaus, $\mathrm{da} \beta$

$$
4-A-2=0 \text {. }
$$

Nimmt man folglich $A=2$, so läßt sich ohne Schwistrigkeid tandhprïfen, daß nach (9.1) und (9.7)-(9.9) allo Ko(ffiziention. von. $)^{M}, M \cdot 768$, gleich Null sind.

SATZ 2a. Es sei $n=2^{\alpha} 3^{\beta} u,(u, 6)=1, M=24$. . Datul is

$$
r(n)=\left\{\begin{array}{lll}
2 \sigma(u)+2 v(M) & \text { für } & \alpha=0, \\
4 \sigma(u) & \text { für } & \alpha=1, \\
4\left(2^{\alpha}-3\right) \sigma(u) . & \text { für } & \alpha>1,
\end{array}\right.
$$

wo $v(M)$ den Koeffizienten von $Q^{M}$ in der Entwioklung der Funktion $\vartheta_{01}(\tau ; 0,12) \vartheta_{41}^{2}(\tau ; 0,12) \vartheta_{81}(\tau ; 0,12)$ nach Potenzen von $Q$ bezeichnet.

Bemerkung. In den von Kloosterman gegebenen Formeln für $r(n)$ tritt statt $\nu(M)$ der Koeffizient $\zeta_{n}$ von $q^{n}$ in $\frac{1}{6} \vartheta_{2}(0 \mid \tau) \vartheta_{1}^{2}\left(\frac{1}{3} \mid \tau\right) \vartheta_{2}\left(\frac{1}{3} \mid \tau\right)$ auf, wobei $q=Q^{24}$ und die $\vartheta_{j}(z \mid \tau)$.Jacobische Thetafunktionen sind.

Beweis. Aus (9.8) folgt

$\vartheta_{01}(\tau ; 0,12) \vartheta_{41}^{2}(\tau ; 0,12) \vartheta_{81}(\tau ; 0,12)$

$$
=Q^{24} \sum_{k=-\infty}^{\infty}(-1)^{k} Q^{24 \cdot 6 k^{2}}\left(\sum_{k=-\infty}^{\infty}(-1)^{k} Q^{24 k(6 k+2)}\right)^{2}\left(\sum_{k=-\infty}^{\infty}(-1)^{k} Q^{24 k(6 k+4)}\right) .
$$

$\vartheta_{01}(\tau ; 0,12) \vartheta_{41}^{2}(\tau ; 0,12) \vartheta_{81}(\tau ; 0,12)$ ist also eine ungerade Funktion von $Q^{24}$, d. h. es ist

$$
\nu(M)=0 \text { für gerade } n .
$$
tung.

§ 10. In diesem Paragraphen wird die Darstellung der Zahlen durch die Form

$$
x_{1}^{2}+x_{2}^{2}+7 x_{3}^{2}+7 x_{4}^{2}
$$

untersucht.

Nach Hilfssatz 14 ist jeder der beiden Zweige

$(10.1) \quad X(\tau)=\left\{\vartheta_{20}(\tau ; 0,2) \vartheta_{00}^{2}(\tau ; 0,2) \vartheta_{01}(\tau ; 0,2) \vartheta_{14}(\tau ; 0,14) \vartheta_{00}^{2}(\tau ; 0,14) \times\right.$

$$
\left.\times \vartheta_{01}(\tau ; 0,14)\right\}^{1 / 2}
$$

eine eindeutige Funktion von $\tau$.

Hufssatz 33. Bei geeignetem $A$ ist die Funktion

$(10.2) \quad \psi(\tau ; 1,7)=\vartheta_{00}^{2}(\tau ; 0,2) \vartheta_{00}^{2}(\tau, 0,14)-\Theta(\tau ; 7)-A X(\tau)$

eine ganze Modulform der Stufe 28 und der Dimension -2.

Der Beweis verläuft ähnlich, wie bei Hilfssatz 31 .

SATZ 3. Wird der Zweig (10.1) durch

$$
\lim _{\operatorname{Im} \tau \rightarrow \infty} e(-\tau) X(\tau)=2
$$

festgelegt, so ist

$$
\vartheta_{00}^{2}(\tau ; 0,2) \vartheta_{00}^{2}(\tau ; 0,14)=\Theta(\tau ; 7)+\frac{4}{3} X(\tau) .
$$




\section{$\mathrm{icm}$}

Beweis. Nach den Hilfssätzen 33 und 1 ist die Funktion $\psi(\tau ; 1,7)$ den indamentalbereich mehr als

$$
\frac{2}{24} 28^{3} \prod_{p \mid 28}\left(1-p^{-2}\right)=1344
$$

Nullstellen hat. Dazu reicht es hin, daß $\tau=i \infty$ ein mohr als 1.344 - facher Null gezeigt werden, daß bei geeigneter: Wahl voll $A$ in Nullpunkt sei. Es soll gezeigt werden, das ben gon $Q$ die Glieder mit $Q^{M}$, $M \leqslant 1344$, verschwinden.

Setzt man in den Hilfssätzen 28, 29 und 30

$$
a=1, \quad a^{\prime}=7, \quad \gamma=0, \quad b=1, \quad v=7^{\beta}, \quad l \cdots 1,
$$

d. h.

$$
M=28 n, \quad n=2^{a} m=2^{u} 7^{\beta} u, \quad u=\prod_{\substack{p ! n \\ p+14}} p^{\beta},
$$

so ergibt sich

$$
\varrho(M)=\frac{2^{\alpha-1}}{3} 7^{\beta+1} \sigma(u) \chi_{2} \chi_{7}
$$

Hierbei ist

$$
\chi_{2}=\left\{\begin{array}{lll}
1 & \text { für } & \alpha=0, \\
2^{-a}\left(2^{\alpha+1}-3\right) & \text { für } & \alpha>0
\end{array}\right.
$$

und

$$
\chi_{7}= \begin{cases}\frac{8}{7} & \text { für } \quad \beta=0, \\ 8 \cdot 7^{-\beta-1} & \text { für } \quad \beta>0,\end{cases}
$$

also in beiden Fällen.

$$
\chi_{7}=8 \cdot 7^{\cdots \cdots-1} \text {. }
$$

Aus (10.4)-(10.6) folgt

$$
\varrho(M)=\left\{\begin{array}{lll}
\frac{4}{3} \sigma(u) & \text { fǜl: } & \alpha=0, \\
\frac{4}{3}\left(2^{\alpha+1}-3\right) \sigma(u) & \text { fiür } & \alpha>0 .
\end{array}\right.
$$

Setzt man $Q=e\left(\frac{\tau}{28}\right)$, so ergibt sich aus (1.4)

$$
\vartheta_{00}^{2}(\tau ; 0,2) \vartheta_{00}^{2}(\tau ; 0,14)=\left(\sum_{k=-\infty}^{\infty} Q^{28 k^{2}}\right)^{2}\left(\sum_{k=-\infty}^{\infty} Q^{196 k^{2}}\right)^{2}
$$

$=1+4 Q^{28}+4 Q^{56}+4 Q^{112}+8 Q^{140}+4 Q^{196}+20 Q^{224}+$

$+20 Q^{252}+8 Q^{280}+16 Q^{308}+32 Q^{336}+8 Q^{364}+$

$+4 Q^{392}+32 Q^{420}+36 Q^{448}+40 Q^{476}+20 Q^{504}+$

$+32 Q^{532}+40 Q^{560}+16 Q^{616}+32 Q^{644}+64 Q^{672}+$

$+28 Q^{700}+8 Q^{728}+64 Q^{756}+4 Q^{784}+24 Q^{812}+$

$+32 Q^{840}+32 Q^{868}+84 Q^{896}+64 Q^{924}+40 Q^{952}+$

$+8 Q^{980}+84 Q^{1008}+56 Q^{1036}+32 Q^{1064}+96 Q^{1092}+$

$+104 Q^{1120}+72 Q^{1148}+80 Q^{1204}+80 Q^{1232}+$

$+104 Q^{1260}+32 Q^{1288}+32 Q^{1316}+160 Q^{1344}+\ldots$,

$X^{2}(\tau)=\sum_{k=-\infty}^{\infty} Q^{7(2 k+1)^{2}}\left(\sum_{k=-\infty}^{\infty} Q^{28 k^{2}}\right)^{2} \sum_{k=-\infty}^{\infty}(-1)^{k} Q^{28 k^{2}} \times$

$$
\begin{gathered}
\times \sum_{k=-\infty}^{\infty} Q^{49(2 k+1)^{2}}\left(\sum_{k=-\infty}^{\infty} Q^{196 k^{2}}\right)^{2} \sum_{k=-\infty}^{\infty}(-1)^{k} Q^{196 k^{2}} \\
=4 Q^{56}\left(1+2 Q^{28}-3 Q^{56}-6 Q^{84}+2 Q^{112}-Q^{168}+12 Q^{196}+4 Q^{224}-\right. \\
-4 Q^{252}-2 Q^{280}-2 Q^{308}-7 Q^{336}-16 Q^{364}+17 Q^{392}+4 Q^{420}+Q^{448}+ \\
+38 Q^{476}-24 Q^{504}-14 Q^{532}-16 Q^{560}-32 Q^{588}+18 Q^{616}-12 Q^{644}+ \\
+24 Q^{672}-4 Q^{700}+21 Q^{728}+60 Q^{756}-64 Q^{784}+12 Q^{812}+7 Q^{840}- \\
-48 Q^{868}+62 Q^{896}+42 Q^{924}+21 Q^{952}-20 Q^{980}-34 Q^{1008}+ \\
+40 Q^{1036}-64 Q^{1064}-4 Q^{1092}-14 Q^{1120}-108 Q^{1148}+40 Q^{1176}- \\
\left.-30 Q^{1204}-16 Q^{1232}-66 Q^{1288}+\ldots\right),
\end{gathered}
$$

woraus

(10.9) $X(\tau)=2 Q^{28}+2 Q^{56}-4 Q^{84}-2 Q^{112}-4 Q^{168}+2 Q^{196}+2 Q^{224}+2 Q^{252}+$

$$
\begin{aligned}
& +4 Q^{336}-8 Q^{364}+2 Q^{392}-2 Q^{448}+12 Q^{476}+2 Q^{504}+4 Q^{532}- \\
& -4 Q^{588}-4 Q^{672}-10 Q^{700}-8 Q^{728}+8 Q^{756}-2 Q^{784}-12 Q^{812}- \\
& -8 Q^{868}+2 Q^{896}+12 Q^{952}-2 Q^{1008}+4 Q^{1036}+4 Q^{1064}+16 Q^{1092}+ \\
& +12 Q^{1148}-4 Q^{1176}+16 Q^{1204}-24 Q^{1316}+4 Q^{1344}+\ldots
\end{aligned}
$$

Berechnet man die Werte von $\varrho(M)$ mittels der Formeln (10.7) und setzt sie in (4.7) ein, so bekommt man 
(10.10) $\Theta(\tau ; 7)=1+\frac{4}{3} Q^{28}+\frac{4}{3} Q^{56}+\frac{16}{3} Q^{84}+\frac{20}{3} Q^{112}+8 Q^{140}+\frac{16}{3} Q^{168}+\frac{4}{3} Q^{196}+$

$$
\begin{aligned}
& +\frac{52}{3} Q^{224}+\frac{52}{3} Q^{252}+8 Q^{280}+16 Q^{308}+\frac{80}{3} Q^{336}+\frac{56}{3} Q^{364}+ \\
& +\frac{4}{3} Q^{392}+32 Q^{420}+\frac{116}{3} Q^{448}+24 Q^{476}+\frac{52}{3} Q^{504}+\frac{80}{3} Q^{532}+ \\
& +40 Q^{500}+\frac{16}{3} Q^{588}+16 Q^{616}+32 Q^{644}+\frac{208}{3} Q^{672}+\frac{124}{3} Q^{700}+ \\
& +\frac{56}{3} Q^{728}+\frac{160}{3} Q^{756}+\frac{20}{3} Q^{784}+40 Q^{812}+32 Q^{840}+\frac{128}{3} Q^{888}+ \\
& +\frac{244}{3} Q^{896}+64 Q^{024}+24 Q^{952}+8 Q^{980}+\frac{260}{3} Q^{1008}+\frac{152}{3} Q^{1036}+ \\
& +\frac{80}{3} Q^{1064}+\frac{224}{3} Q^{1092}+104 Q^{1120}+56 Q^{1148}+{ }^{16} \frac{16}{3} Q^{1176}+ \\
& +\frac{176}{3} Q^{1204}+80 Q^{1232}+104 Q^{1200}+32 Q^{1288}+64 Q^{1310}+ \\
& +\frac{464}{3} Q^{1344}+\ldots
\end{aligned}
$$

Man wähle jetzt die Konstante $A$ so, daß der Koelfizienti von. $\left(^{28}\right.$ in der Entwicklung der Funktion $\psi(\tau ; 1,7)$ verschwindet. Nach (1.0.2), (10.8)-(10.10) kommt dies darauf hinaus, daß

$$
4-2 A-\frac{4}{3}=0 \text {. }
$$

Nimmt man folglich $A=\frac{4}{3}$, so läßt sich ohne Schwierigkeiti nachprüfen, daß nach (10.2) und (10.8)-(10.10) alle Koeffizienten von. $Q^{M}$, $M \leqslant 1344$, gleich Null sind.

SATZ 3a. Es sei $n=2^{\alpha} 7^{\beta} u,(u, 14)=1, M$. $=28 n$. Inann ist

$$
r(n)=\left\{\begin{array}{lll}
\frac{4}{3} \sigma(u)+\frac{4}{3} \nu(M) & \text { für } & \alpha=0, \\
\frac{4}{3}\left(2^{\alpha+1}-3\right) \sigma(u)+\frac{4}{3} \nu(M) & \text { für } & \alpha>0,
\end{array}\right.
$$

wo $v(M)$ den Koeffizienten von $Q^{M}$ in der Entwioklung der F'unktion $X(\tau)$ nach Potenzen von $Q$ bezeichnet.

Bemerkung. In den von Kloosterman gegebenen Formeln für $r(n)$ tritt statt $\nu(M)$ der Kooffizient $v_{n}$ von $q^{n}$ in $\left\{\vartheta_{2}(0 \mid \tau) \vartheta_{3}^{2}(0 \mid \tau) v_{4}(0 \mid \tau) \times\right.$ $\left.\times \vartheta_{2}(0 \mid 7 \tau) \vartheta_{3}^{2}(0 \mid 7 \tau) \vartheta_{4}(0 \mid 7 \tau)\right\}^{1 / 2}$ auf, wobei $q=Q^{28}$ und dis $\vartheta_{j}(0 \mid \tau)$ Jacobisehe Thetafunktionen sind. (10.7).

Beweis. Die Behauptung folgt ans (10.3), (4.2), (4.3), (1.7) und

§ 11. In diesem Paragraphen wird die Darstellung der zahlon dureh. die Form

untersucht.

$$
x_{1}^{2}+x_{2}^{2}+9 x_{3}^{2}+9 x_{4}^{2}
$$

HILFssatz 34. Die Funktion

(11.1) $\quad \psi(\tau ; 1,9)=\vartheta_{00}^{2}(\tau ; 0,2) \vartheta_{00}^{2}(\tau ; 0,18)-\Theta(\tau ; 9)-A \vartheta_{61}^{4}(\tau ; 0,18)$

ist bei konstantem A eine ganze Modulform der Stufe 36 und der Dimension -2 .

Der Beweis verläuft ähnlich, wie bei dem Hilfssatz 31 .

SATZ 4.

$$
\vartheta_{00}^{2}(\tau ; 0,2) \vartheta_{00}^{2}(\tau ; 0,18)=\Theta(\tau ; 9)+\frac{8}{3} \vartheta_{61}^{4}(\tau ; 0,18) .
$$

Beweis. Nach den Hilfssätzen 34 und 1 ist die Funktion $\psi(\tau ; 1,9)$ identisch Null, wenn sie in ihrem Fundamentalbereich mehr als

$$
\frac{2}{24} 36^{3} \prod_{p \mid 36}\left(1-p^{-2}\right)=2592
$$

Nullstellen hat. Dazu reicht es hin, daß $\tau=i \infty$ ein mehr als 2592 -facher Nullpunkt sei. Es soll gezeigt werden, daß bei geeigneter Wahl von $A$ in der Entwicklung von $\psi(\tau ; 1,9)$ nach Potenzen von $Q$ die Glieder mit $Q^{M}$, $M \leqslant 2592$ verschwinden.

Setzt man in den Hilfssätzen 28, 29 und 30

$$
a=1, \quad a^{\prime}=9, \quad \gamma=0, \quad b=1, \quad v=3^{\beta}, \quad l=2,
$$

so ergibt sich

$$
M=36 n, \quad n=2^{a} \cdot m=2^{a} 3^{\beta} u, \quad u=\prod_{\substack{p \mid n \\ p \nmid 6}} p^{\beta},
$$

Hierbei ist

und

$$
\varrho(M)=2^{\alpha} 3^{\beta} \sigma(u) \chi_{2} \chi_{3} .
$$

$$
\chi_{2}=\left\{\begin{array}{lll}
1 & \text { für } & \alpha=0 \\
3 \cdot 2^{-a} & \text { für } & \alpha>0
\end{array}\right.
$$

$$
\chi_{3}=\left\{\begin{array}{lll}
\frac{4}{3} & \text { für } & \beta=0 \\
0 & \text { für } & \beta=1 \\
\frac{4}{3}\left(1-3^{-\beta+1}\right) & \text { für } & \beta>1
\end{array}\right.
$$

also

$$
\chi_{3}=\left\{\begin{array}{lll}
\frac{4}{3} & \text { für } & \beta=0 \\
\frac{4}{3}\left(1-3^{-\beta+1}\right) & \text { für } & \beta>0 .
\end{array}\right.
$$


Aus (11.3)-(11.5) folgt

$$
\varrho(M)=\left\{\begin{array}{lll}
12\left(3^{\beta-1}-1\right) \sigma(u) & \text { für } & \alpha>0, \beta>0, \\
4\left(3^{\beta-1}-1\right) \sigma(u) & \text { für } & \alpha=0, \beta>0, \\
4 \sigma(u) & \text { für } & \alpha>0, \beta=0, \\
\frac{4}{3} \sigma(n) & \text { für } & \alpha=\beta=0 .
\end{array}\right.
$$

Setzt $\operatorname{man} Q=e\left(\frac{\tau}{36}\right)$, so ergibt sich aus (1.4)

$$
\begin{aligned}
& \vartheta_{00}^{2}(\tau ; 0,2) \vartheta_{00}^{2}(\tau ; 0,18)=\left(\sum_{l=-\infty}^{\infty} Q^{36 k^{2}}\right)^{2}\left(\sum_{k=\pi n-\infty}^{\infty} Q^{324 h k^{2}}\right)^{2} \\
& =1+4 Q^{36}+4 Q^{72}+4 Q^{144}+8 Q^{180}+4 \cdot Q^{288}+8 Q^{324}+ \\
& +24 Q^{360}+16 Q^{396}+24 Q^{408}+32 Q^{504}+4 Q^{576}+ \\
& +24 Q^{612}+24 Q^{648}+48 Q^{684}+24 Q^{720}+48 Q^{702}+ \\
& +32 Q^{828}+28 Q^{900}+56 Q^{936}+32 Q^{072}+32 Q^{1008}+ \\
& +40 Q^{1044}+32 Q^{1116}+4 Q^{1152}+72 Q^{1224}+64 Q^{1200}+ \\
& +24 Q^{1296}+24 Q^{1332}+80 Q^{1368}+24 Q^{1440}+ \\
& +56 Q^{1476}+80 Q^{1548}+48 Q^{1584}+48 Q^{1620}-1 \\
& +96 Q^{1056}+64 Q^{1002}+1000 Q^{1764}+124 Q^{1800} \text { - } \\
& +56 Q^{1872}+72 Q^{1008}+96 Q^{1044}+96 Q^{1980}+32 Q^{2010}+ \\
& +120 Q^{2088}+80 Q^{2124}+120 Q^{2106}+128 Q^{2232}+ \\
& +64 Q^{2288}+4 Q^{2304}+112 Q^{2340}+48 Q^{2412}+ \\
& +72 Q^{2448}+96 Q^{2484}+192 Q^{2520}+96 Q^{2550}+ \\
& +24 Q^{2592}+\ldots \text {, } \\
& =Q^{36}-4 Q^{252}+2 Q^{408}+8 Q^{684} \ldots 5 Q^{900}-4 Q^{1116}-10 Q^{1334}+ \\
& +8 Q^{1548}+9 Q^{1704}+1.4 Q^{2106}-16 Q^{2412}-14 Q^{2024}+\ldots
\end{aligned}
$$

Berechnet man die Werte von $\varrho(. M)$ mittels der Formeln (1.1.6) und setzt sie in (4.7) ein, so bekommt man.

(11.10) $\Theta(\tau ; 9)=1+\frac{4}{3} Q^{36}+4 Q^{72}+4 Q^{144}+8 Q^{180}++^{32} Q^{252}+4 Q^{288}+8 Q^{324}+$

$$
+24 Q^{360}+16 Q^{396}+\frac{56}{3} Q^{488}+32 Q^{504}+4 Q^{576}+24 Q^{012}+
$$

$$
\begin{aligned}
& +24 Q^{648}+\frac{80}{3} Q^{684}+24 Q^{720}+48 Q^{792}+32 Q^{828}+\frac{124}{3} Q^{900}+ \\
& +56 Q^{936}+32 Q^{972}+32 Q^{1008}+40 Q^{1044}+\frac{128}{3} Q^{1116}+ \\
& +4 Q^{1152}+72 Q^{1224}+64 Q^{1260}+24 Q^{1296}+\frac{152}{3} Q^{1382}+80 Q^{1368}+ \\
& +24 Q^{1440}+56 Q^{1476}+\frac{176}{3} Q^{1548}+48 Q^{1584}+48 Q^{1620}+ \\
& +96 Q^{1656}+64 Q^{1692}+76 Q^{1764}+124 Q^{1800}+56 Q^{1872}+ \\
& +72 Q^{1908}+96 Q^{1944}+96 Q^{1980}+32 Q^{2016}+120 Q^{2088}+ \\
& +80 Q^{2124}+\frac{248}{3} Q^{2196}+128 Q^{2232}+64 Q^{2268}+4 Q^{2304}+ \\
& +112 Q^{2340}+\frac{272}{3} Q^{2412}+72 Q^{2448}+96 Q^{2484}+192 Q^{2520}+ \\
& +96 Q^{2556}+24 Q^{2592}+\ldots
\end{aligned}
$$

Man wähle jetzt die Konstante $A$ so, daß der Koeffizient von $Q^{36}$ in der Entwicklung der Funktion $\psi(\tau ; 1,9)$ verschwindet. Nach (11.1), (11.7)-(11.10) kommt dies darauf hinaus, daß

$$
4-A-\frac{4}{3}=0 .
$$

Nimmt man folglich $A=\frac{8}{3}$, so läßt sich ohne Schwierigkeit nachprüfen, daß nach (11.1) und (11.7)-(11.10) alle Koeffizienten von $Q^{M}$, $M \leqslant 2592$ gleich Null sind.

SAtz 4a. Es sei $n=2^{a} 3^{\beta} u,(u, 6)=1, M=36 n$. Dann ist

$$
r(n)=\left\{\begin{array}{lll}
12\left(3^{\beta-1}-1\right) \sigma(u) & \text { für } & \alpha>0, \quad \beta>0, \\
4\left(3^{\beta-1}-1\right) \sigma(u) & \text { für } & \alpha=0, \quad \beta>0, \\
4 \sigma(u) & \text { für } & \alpha>0, \quad \beta=0, \\
\frac{4}{3} \sigma(n)+\frac{8}{3} \nu(M) & \text { für } & n \equiv 1(\bmod 6), \\
\frac{4}{3} \sigma(n) & \text { für } & n \equiv 5(\bmod 6),
\end{array}\right.
$$

wo $\nu(M I)$ den Koeffizienten von $Q^{M}$ in der Entwicklung der Funktion $\vartheta_{61}^{4}(\tau ; 0,18)$ nach Potenzen von $Q$ bezeichnet.

Bemerkung. In dieser Formel ist nur die vierte Zeile neu; in den übrigen Fällen wurde sie auf elementarem Wege von Liouville [6] erhalten, der seinen Beweis nicht veröffentlicht hat.

Beweis. Aus (11.8) folgt

$$
\vartheta_{61}^{4}(\tau ; 0,18)=Q^{36}\left(\sum_{k=-\infty}^{\infty}(-1)^{k} Q^{36 \cdot 3 k(3 k+1)}\right)^{4} .
$$


Die Exponenten in der Entwicklung der Funktion $\vartheta_{61}^{4}(\tau ; 0,18)$ nach Po tenzen von $Q$ haben daher die Gestalt

$$
M=36 n=36(6 h+1),
$$

d. h. es ist

$$
\nu(M)=0 \quad \text { für } \quad n \neq 1(\bmod 6) .
$$

Aus (11.2), (4.2), (4.3), (4.7), (11.6) und (11.12) ergibt sich dio Bohauptung.

§ 12. In diesem Paragraphen wird dio Darstellung der Zahlen, dureh die Form

$$
x_{1}^{2}+x_{2}^{2}+10 x_{3}^{2}+10 x_{4}^{2}
$$

untersucht.

Hinfssatz 35. Die Funtion

(12.1) $\quad \psi(\tau ; 1,10)=\vartheta_{00}^{2}(\tau ; 0,2) \vartheta_{00}^{2}(\tau ; 0,20)-\Theta(\tau ; 10)-$

$-A_{1} \vartheta_{21}^{2}(\tau ; 0,10) \vartheta_{61}^{2}(\tau ; 0,10)-A_{2} \vartheta_{00}^{2}(\tau ; 0,4) \vartheta_{40}(\tau ; 0,20) \vartheta_{12,0}(\tau ; 0,20)-$

$-A_{3} \vartheta_{41}^{2}(\tau ; 0,20) \vartheta_{12,1}^{2}(\tau ; 0,20)-A_{4} \vartheta_{20,0}^{2}(\tau ; 0,20) \vartheta_{80}(\tau ; 0,20) \vartheta_{16,0}(\tau ; 0,20)$

ist bei konstanten $A_{1}, A_{2}, A_{3}, A_{4}$ eine ganze Modulform der stufe 40 und der Dimension -2 .

Der Beweis verläuft ähnlich, wie bei Hilfssatz 31 .

SATz 5.

(12.2) $\quad \vartheta_{00}^{2}(\tau ; 0,2) \vartheta_{00}^{2}(\tau ; 0,20)=\Theta(\tau ; 10)+$ $+\frac{8}{3} \vartheta_{21}^{2}(\tau ; 0,10) \vartheta_{61}^{2}(\tau ; 0,10)+\frac{2}{3} \vartheta_{00}^{2}(\tau ; 0,4) \vartheta_{10}(\tau ; 0,20) \vartheta_{12,0}(\tau ; 0,20)+$

$+\frac{8}{3} \vartheta_{41}^{2}(\tau ; 0,20) \vartheta_{12,1}^{2}(\tau ; 0,20)-\frac{10}{3} \vartheta_{20,0}^{2}(\tau ; 0,20) \vartheta_{80}(\tau ; 0,20) \vartheta_{16,0}(\tau ; 0,20)$.

Beweis. Nach den Hilfssätzen 35 und 1 ist die Funktion $\psi(\tau ; 1,10)$ identisch Null, wenn sie in ihrem Fundamentalbereich mehr als

$$
\frac{2}{24} 40^{3} \prod_{p \mid 40}\left(1-p^{-2}\right)=3840
$$

Nullstellen hat. Dazu reicht es hin, daß $\tau=i \infty$ ein mohr als 384:0 facher Nullpunkt sei. Es soll gezeigt werden, daß bei geeigneter Wahl von. $A_{1}, A_{x}$, $A_{\mathbf{8}}, A_{\mathbf{4}}$ in der Entwicklung von $\psi(\tau ; 1,10)$ nach Potenzen von $Q$ dio Glieder mit $Q^{M}, M \leqslant 3840$ verschwinden.

Setzt man in den Hilfssätzen 28, 29 und 30

$$
a=10, \quad a^{\prime}=1, \quad \gamma=1, \quad b=5, \quad v=55^{\beta}, \quad l=1,
$$

d. h.

$$
M=40 n, \quad n=2^{\alpha} m=2^{\alpha} 5^{\beta} u, \quad u=\prod_{\substack{p \mid n \\ p \times 10}} p^{\beta},
$$

so ergibt sich

Hierbei ist

und

$$
\varrho(M)=\frac{2^{\alpha-1}}{3} 5^{\beta+1} \sigma(u) \chi_{2} \chi_{5} .
$$

$$
\chi_{2}=\left\{\begin{array}{lll}
1 & \text { für } & \alpha=0,1, \\
3 \cdot 2^{-\alpha+1} & \text { für } & \alpha>1
\end{array}\right.
$$

Aus (12.3)-(12.5) folgt

$$
\varrho(M)=\left\{\begin{array}{lll}
\frac{2^{\alpha}}{3}\left(5^{\beta+1}-3\right) \sigma(u) & \text { für } & \alpha=0,1, \\
2\left(5^{\beta+1}-3\right) \sigma(u) & \text { für } & \alpha>1 .
\end{array}\right.
$$

Setzt $\operatorname{man} Q=e\left(\frac{\tau}{40}\right)$, so ergibt sich aus (1.4)

$$
\begin{aligned}
& \vartheta_{00}^{2}(\tau ; 0,2) \vartheta_{00}^{2}(\tau ; 0,20)=\left(\sum_{k=-\infty}^{\infty} Q^{40 k^{2}}\right)^{2}\left(\sum_{k=-\infty}^{\infty} Q^{400 k^{2}}\right)^{2} \\
(12.7)= & 1+4 Q^{40}+4 Q^{80}+4 Q^{160}+8 Q^{200}+4 Q^{320}+4 Q^{360}+12 Q^{400}+16 Q^{440}+ \\
+ & 16 Q^{480}+8 Q^{520}+16 Q^{560}+32 Q^{600}+4 Q^{640}+8 Q^{680}+20 Q^{720}+ \\
+ & 16 Q^{760}+44 Q^{800}+16 Q^{840}+16 Q^{880}+32 Q^{920}+16 Q^{960}+44 Q^{1000}+ \\
+ & 24 Q^{1040}+32 Q^{1080}+32 Q^{1120}+24 Q^{1160}+64 Q^{1200}+4 Q^{1280}+ \\
+ & 32 Q^{1320}+8 Q^{1360}+48 Q^{1400}+52 Q^{1440}+40 Q^{1480}+16 Q^{1520}+ \\
+ & 32 Q^{1560}+44 Q^{1600}+24 Q^{1640}+32 Q^{1680}+48 Q^{1760}+88 Q^{1800}+48 Q^{1840}+ \\
+ & 32 Q^{1880}+16 Q^{1920}+52 Q^{1960}+84 Q^{2000}+64 Q^{2040}+56 Q^{2080}+ \\
+ & 40 Q^{2120}+64 Q^{2160}+96 Q^{2200}+32 Q^{2240}+64 Q^{2280}+56 Q^{2320}+ \\
+ & 48 Q^{2360}+176 Q^{2400}+40 Q^{2440}+32 Q^{2480}+96 Q^{2520}+4 Q^{2560}+ \\
+ & 96 Q^{2600}+64 Q^{2640}+64 Q^{2680}+72 Q^{2720}+48 Q^{2760}+112 Q^{2800}+ \\
+ & 32 Q^{2840}+52 Q^{2880}+40 Q^{2920}+56 Q^{2960}+160 Q^{3000}+80 Q^{3040}+ \\
+ & 32 Q^{3080}+64 Q^{3120}+64 Q^{3160}+44 Q^{3200}+84 Q^{3840}+72 Q^{3280}+ \\
+ & 32 Q^{3320}+128 Q^{3360}+144 Q^{3400}+32 Q^{3440}+64 Q^{3480}+48 Q^{3520}+ \\
+ & 40 Q^{3560}+188 Q^{3600}+96 Q^{3640}+96 Q^{3680}+96 Q^{3720}+48 Q^{3760}+ \\
+ & 160 Q^{3800}+16 Q^{3840}+\ldots,
\end{aligned}
$$


(12.8) $\vartheta_{21}^{2}(\tau ; 0,10) \vartheta_{61}^{2}(\tau ; 0,10)=\left(\sum_{k=-\infty}^{\infty}(-1)^{k} Q^{2(10 k+1)^{2}}\right)^{2}\left(\sum_{k=-\infty}^{\infty}(-1)^{k} Q^{2(10 k+3)^{2}}\right)^{2}$

$(12.9)=Q^{40}-2 Q^{120}-Q^{200}+2 Q^{280}+Q^{360}+2 Q^{520}+2 Q^{600}-6 Q^{680}-$

$-4 Q^{760}-4 Q^{840}+6 Q^{920}+Q^{1000}+4 Q^{1080}+6 Q^{1160}-4 Q^{1240}-2 Q^{1400}+$ $+2 Q^{1480}-4 Q^{1500}+6 Q^{1640}-10 Q^{1720}-Q^{1800}-6 Q^{1880}-3 Q^{1000}+$

$+12 Q^{2040}-6 Q^{2120}+8 Q^{2280}+12 Q^{2300}+2 Q^{2440}+2 Q^{2520}-2 Q^{2000}+$ $+2 Q^{2680}-12 Q^{2780}-12 Q^{2840}+2 Q^{2920}-2 Q^{3000}+8 Q^{3100}-11 Q^{3240}+$ $+6 Q^{3320}+6 Q^{3400}-12 Q^{3480}-6 Q^{3660}+4 Q^{3640}+8 Q^{3720}+4 Q^{3800}+1$ $+4 Q^{3880}+\ldots$

$\vartheta_{00}^{2}(\tau ; 0,4) \vartheta_{40}(\tau ; 0,20) \vartheta_{12,0}(\tau ; 0,20)$

$(12.10)=\left(\sum_{k=-\infty}^{\infty} Q^{80 \kappa^{2}}\right)^{2} \sum_{k=-\infty}^{\infty} Q^{(20 k+2)^{2}} \sum_{t=-\infty}^{\infty} Q^{(20 \% t+6)^{2}}$

$(12.11)=Q^{40}+4 Q^{120}+5 Q^{200}+4 Q^{280}+9 Q^{360}+12 Q^{440}+10 Q^{520}+16 Q^{600}+$ $+18 Q^{880}+20 Q^{760}+28 Q^{840}+20 Q^{920}+21 Q^{1000}+32 Q^{1080}+22 Q^{1160}+$ $+24 Q^{1240}+40 Q^{1320}+32 Q^{1400}+34 Q^{1480}+48 Q^{1560}+30 Q^{1040}+$ $+36 Q^{1720}+53 Q^{1800}+44 Q^{1880}+53 Q^{1960}+56 Q^{2040}+50 Q^{2120}+$ $+52 Q^{2200}+64 Q^{2280}+44 Q^{2360}+50 Q^{2440}+92 Q^{2520}+58 Q^{2000} \ldots$. $+60 Q^{2880}+84 Q^{2780}+64 Q^{2840}+58 Q^{2020}+84 Q^{3000}+72 Q^{3080}+$ $+64 Q^{3160}+109 Q^{3240}+60 Q^{3320}+74 Q^{3400}+104 Q^{3480}+74 Q^{3560}+$ $+96 Q^{3640}+104 Q^{3720}+84 Q^{3800}+\ldots$,

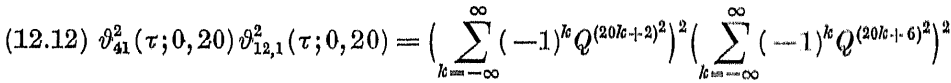

$(12.13)=Q^{80}-2 Q^{240}-Q^{400}+2 Q^{500}+Q^{720}+2 Q^{1040}+2 Q^{1200} \ldots 6 Q^{1300}$ $-4 Q^{1520}-4 Q^{1880}+6 Q^{1840}+Q^{2000}+4 Q^{2160}+1+6 Q^{2820}-4 Q^{24860}$ $-2 Q^{2800}+2 Q^{2060}-4 Q^{3120}+6 Q^{3280}-10 Q^{3440}-Q^{3000}-6 Q^{3700}+\ldots$, $\vartheta_{20,0}^{2}(\tau ; 0,20) \vartheta_{30}(\tau ; 0,20) \vartheta_{16,0}(\tau ; 0,20)$ $(12.14)=\left(\sum_{k=-\infty}^{\infty} Q^{(20 k+10)^{2}}\right)^{2} \sum_{k=-\infty}^{\infty} Q^{(20 k+4)^{2}} . \sum_{k=-\infty}^{\infty} Q^{(20 k t+2)^{2}}$
$(12.15)=4 Q^{280}+4 Q^{360}+4 Q^{520}+4 Q^{600}+4 Q^{840}+4 Q^{920}+4 Q^{1000}+8 Q^{1080}+$ $+8 Q^{1160}+8 Q^{1240}+8 Q^{1320}+8 Q^{1400}+4 Q^{1480}+8 Q^{1560}+12 Q^{1640}+$ $+8 Q^{1720}+12 Q^{1800}+4 Q^{1880}+4 Q^{1960}+16 Q^{2040}+4 Q^{2120}+8 Q^{2200}+$ $+16 Q^{2280}+16 Q^{2360}+12 Q^{2440}+12 Q^{2520}+12 Q^{2600}+8 Q^{2680}+$ $+12 Q^{2760}+8 Q^{5840}+16 Q^{2920}+16 Q^{3000}+24 Q^{3080}+16 Q^{3160}+$ $+12 Q^{3240}+24 Q^{3320}+16 Q^{3400}+16 Q^{3480}+16 Q^{3560}+16 Q^{3640}+$ $+24 Q^{3720}+16 Q^{3800}+24 Q^{3880}+\ldots$

Berechnet man die Werte von $\varrho(M)$ mittels der Formeln (12.6) und setzt sie in (4.7) ein, so bekommt man

(12.16) $\Theta(\tau ; 10)=1+\frac{2}{3} Q^{40}+\frac{4}{3} Q^{80}+\frac{8}{3} Q^{120}+4 Q^{160}+\frac{22}{3} Q^{200}+\frac{16}{3} Q^{240}+\frac{16}{3} Q^{280}$ $+4 Q^{320}+\frac{26}{3} Q^{360}+\frac{44}{3} Q^{400}+8 Q^{440}+16 Q^{480}+\frac{28}{3} Q^{580}+$ $+\frac{32}{3} Q^{560}+\frac{88}{3} Q^{600}+4 Q^{640}+12 Q^{680}+\frac{52}{3} Q^{720}+\frac{40}{3} Q^{760}+$ $+44 Q^{800}+\frac{64}{3} Q^{840}+16 Q^{880}+16 Q^{920}+16 Q^{960}+\frac{122}{3} Q^{1000}+$ $+\frac{56}{3} Q^{1040}+\frac{80}{3} Q^{1080}+32 Q^{1120}+20 Q^{1160}+\frac{176}{3} Q^{1200}+$ $+\frac{64}{3} Q^{1240}+4 Q^{1280}+32 Q^{1320}+24 Q^{1360}+\frac{176}{3} Q^{1400}+$ $+52 Q^{1440}+\frac{76}{3} Q^{1480}+\frac{80}{3} Q^{1520}+\frac{112}{3} Q^{1560}+44 Q^{1600}+$ $+28 Q^{1640}+\frac{128}{3} Q^{1680}+\frac{88}{3} Q^{1720}+48 Q^{1760}+\frac{286}{3} Q^{1800}+$ $+32 Q^{1840}+32 Q^{1880}+16 Q^{1920}+38 Q^{1960}+\frac{244}{3} Q^{2000}+$ $+48 Q^{2040}+56 Q^{2080}+36 Q^{2120}+\frac{160}{3} Q^{2160}+88 Q^{2200}+$ $+32 Q^{2240}+\frac{160}{3} Q^{2280}+40 Q^{2320}+40 Q^{2360}+176 Q^{2400}+$ $+\frac{124}{3} Q^{2440}+\frac{128}{3} Q^{2480}+\frac{208}{3} Q^{2520}+4 Q^{2560}+\frac{308}{3} Q^{2600}+$ $+64 Q^{2640}+\frac{136}{3} Q^{2680}+72 Q^{2720}+64 Q^{2760}+\frac{352}{3} Q^{2800}+$ $+48 Q^{2840}+52 Q^{2880}+\frac{148}{3} Q^{2920}+\frac{152}{3} Q^{2960}+\frac{488}{3} Q^{3000}+$ $+80 Q^{3040}+64 Q^{3080}+\frac{224}{3} Q^{3120}+\frac{160}{3} Q^{3160}+44 Q^{3200}+$ $+\frac{242}{3} Q^{3240}+56 Q^{3280}+56 Q^{3320}+128 Q^{3360}+132 Q^{3400}+$ $+\frac{176}{3} Q^{3440}+80 Q^{3480}+48 Q^{3520}+60 Q^{3560}+\frac{572}{3} Q^{3600}+$ $+\frac{224}{3} Q^{3640}+96 Q^{3680}+\frac{256}{3} Q^{3720}+64 Q^{3760}+\frac{440}{3} Q^{3800}+$ $+16 Q^{3840}+\ldots$ 
Wähle jetzt die Konstanten $A_{1}, A_{2}$ und $A_{3}$ so, da $B$ die Koeffizienten $Q^{40} Q^{80}$ und $Q^{120}$ in der Entwicklung der Funktion $\psi(\tau ; 1,1.0)$ zerschwinden. Nach (12.1), (12.7), (12.9), (12.11), (12.13) und (12.16) kommt dies darauf hinaus, daß

$$
4-A_{1}-A_{2}-\frac{2}{3}=0, \quad 4-A_{3}-\frac{4}{3}=0, \quad 2 A_{1}-4 A_{2}-\frac{8}{3}=0,
$$

woraus

$$
A_{1}=\frac{8}{3}, \quad A_{2} \quad \frac{2}{3}, \quad A_{3}=\frac{8}{3} .
$$

Die Konstante $A_{4}$ wähle man so, daß der Koeffizienti von $Q^{280}$ in dor Entwicklung der Funktion $\psi(\tau ; 1,1.0)$ verschwindet. Nach (12.1), (12.7), $(12.9),(12.11),(12.13),(12.15)$ und (12.16) kommt dies darauf hinaus, daß

$$
2 A_{1}+4 A_{2}+4 A_{4}+\frac{16}{3}=0,
$$

woraus

$$
A_{4}=-\frac{10}{3} \text {. }
$$

Bestimmt man folglich die Konstanten $A_{1}, A_{2}, A_{3}$ und $A_{4}$ durch (12.17), (12.18), so läßt sich ohne Schwierigkeit nachprüfen, daß nach (12.1), (12.7), (12.9), (12.11), (12.13), (12.15) und (12.16) alle Kooffizionten von $Q^{M}, M \leqslant 3840$ gleich Null sind.

SATz 5a. Es sei $n=2^{a} 5^{\beta} u,(u, 10)=1, M=40 n$. Dann ist

(12.19), $r(n)= \begin{cases}\left.\frac{1}{3}\left(5^{\beta+1}-3\right) \sigma(u)+\frac{8}{3} \nu_{1}(M)+\frac{2}{3} \nu_{2}(M)-{ }_{3}^{10} \nu_{1}(M)\right) & \text { für } \alpha=0, \\ \frac{2}{3}\left(5^{\beta+1}-3\right) \sigma(u)+\frac{8}{3} \nu_{3}(M) & \text { für } \alpha=1, \\ 2\left(5^{\beta+1}-3\right) \sigma(u) & \text { für } \alpha>1,\end{cases}$

wo $v_{1}(M), v_{2}(M), v_{3}(M)$ und $v_{4}(M)$ die Koeffizienten von $Q^{M}$ in den Lntwiokm lungen der Funktionen

$$
\vartheta_{21}^{2}(\tau ; 0,10) \vartheta_{61}^{2}(\tau ; 0,10), \vartheta_{00}^{2}(\tau ; 0,4) \vartheta_{40}(\tau ; 0,20) \vartheta_{12,0}(\tau ; 0,20),
$$

$\vartheta_{41}^{2}(\tau ; 0,20) \vartheta_{12,1}^{2}(\tau ; 0,20)$ und $\vartheta_{20,0}^{2}(\tau ; 0,20) \vartheta_{00}(\tau ; 0,20) \vartheta_{16,0}(\tau ; 0,20)$

nach Potenzen von $Q$ bezeichnen.

Beweis. Aus (12.8) folgt

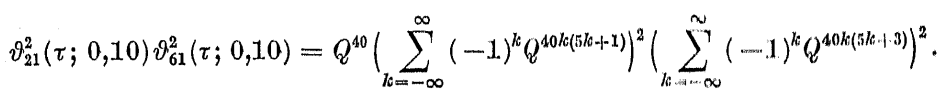

$\vartheta_{21}^{2}(\tau ; 0,10) \vartheta_{61}^{2}(\tau ; 0,10)$ ist also eine ungerade Funktion von $Q^{40}, \mathrm{~d} . \mathrm{h}$. es ist

(12.20)

$$
v_{1}(M)=0 \text { für gerade } n .
$$

Aus (12.10) folgt

$\vartheta_{00}^{2}(\tau ; 0,4) \vartheta_{40}(\tau ; 0,20) \vartheta_{12,0}(\tau ; 0,20)$

d. h.

$$
=Q^{40}\left(\sum_{k=-\infty}^{\infty} Q^{80 k^{2}}\right)^{2} \sum_{k=-\infty}^{\infty} Q^{80 k(5 k+1)} \sum_{k=-\infty}^{\infty} Q^{80 k(5 k+3)}
$$

(12.21)

$$
v_{2}(M)=0 \quad \text { für gerade } n .
$$

Aus (12.12) folgt

$\vartheta_{41}^{2}(\tau ; 0,20) \vartheta_{12,1}^{2}(\tau ; 0,20)=Q^{80}\left(\sum_{k=-\infty}^{\infty}(-1)^{k} Q^{80 k(5 k+1)}\right)^{2}\left(\sum_{k=-\infty}^{\infty}(-1)^{k} Q^{80 k(5 k+3)}\right)^{2}$.

$\vartheta_{41}^{2}(\tau ; 0,20) \vartheta_{12,1}^{2}(\tau ; 0,20)$ ist also eine ungerade Funktion von $Q^{80}$ und eine gerade Funktion von $Q^{40}$, d. h. es ist

(12.22) $\quad \nu_{3}(M)=0$ für ungerade $n$ oder für $n \equiv 0(\bmod 4)$.

Aus (12.14) folgt

$\vartheta_{20}^{2}(\tau ; 0,20) \vartheta_{80}(\tau ; 0,20) \vartheta_{16,0}(\tau ; 0,20)$

d. h.

$$
=Q^{280}\left(\sum_{k=-\infty}^{\infty} Q^{400 k(k+1)}\right)^{2} \sum_{k=-\infty}^{\infty} Q^{80 k(5 k+2)} \sum_{k=-\infty}^{\infty} Q^{80 k(5 k+4)},
$$

$$
v_{4}(M)=0 \quad \text { für gerade } n .
$$

Aus (12.2), (4.2), (4.3), (4.7), (12.6) und (12.20)-(12.23) ergibt sich die Behauptung.

\$ 13. In diesem Paragraphen wird die Darstellung der Zahlen durch die Frorm

$$
2 x_{1}^{2}+2 x_{2}^{2}+5 x_{3}^{2}+5 x_{4}^{2}
$$

untersucht

HufFssatz 36. Die Funktion

(13.1) $\quad \psi(\tau ; 2,5)=\vartheta_{00}^{2}(\tau ; 0,4) \vartheta_{00}^{2}(\tau ; 0,10)-\Theta(\tau ; 10)-$

$-A_{1} \vartheta_{00}^{2}(\tau ; 0,4) \vartheta_{40}(\tau ; 0,20) \vartheta_{12,0}(\tau ; 0,20)-A_{2} \vartheta_{41}^{2}(\tau ; 0,20) \vartheta_{12,1}^{2}(\tau ; 0,20)-$

$$
-\dot{A_{3}} \vartheta_{20,0}^{2}(\tau ; 0,20) \vartheta_{80}(\tau ; 0,20) \vartheta_{16,0}(\tau ; 0,20)
$$


ist bei lonstanten $A_{1}, A_{2}, A_{3}$ eine ganze Modulform der Stufe 40 und der Dimension -2 .

Der Beweis verläuft ähnlich, wie bei Hilfssatz 31 .

SATZ 6.

$$
\begin{aligned}
(13.2) \quad \vartheta_{00}^{2}(\tau ; 0,4) \vartheta_{00}^{2}(\tau ; 0,10) & =\Theta(\tau ; 10)- \\
-\frac{2}{3} \vartheta_{00}^{2}(\tau ; 0,4) \vartheta_{40}(\tau ; 0,20) \vartheta_{12,0}(\tau ; 0,20)+\frac{8}{3} \vartheta_{41}^{2}(\tau ; 0,20) \vartheta_{12,1}^{2}(\tau ; 0,20)+ & +\frac{10}{3} \vartheta_{20,0}^{2}(\tau ; 0,20) \vartheta_{80}(\tau ; 0,20) \vartheta_{16,0}(\tau ; 0,20) .
\end{aligned}
$$

Beweis. Nach den Hilfssätzen 36 und 1 ist die Funktion $\psi(\tau ; 2, \pi)$ identisch Null, wenn bei geeigneter Wahl von $A_{1}, A_{2}, A_{3}$ in der Lntwick lung von $\psi(\tau ; 2,5)$ nach Potenzen von $Q$ die Glieder mit $Q^{M}, M<3840$ verschwinden.

Setzt $\operatorname{man} Q=e\left(\frac{\tau}{40}\right)$, so ergibt sich aus (1.4)

$$
\vartheta_{00}^{2}(\tau ; 0,4) \vartheta_{00}^{2}(\tau ; 0,10)=\left(\sum_{k=-\infty}^{\infty} Q^{80 k^{2}}\right)^{2}\left(\sum_{k=0}^{\infty} Q^{200 k^{2}}\right)^{2}
$$

$$
\begin{aligned}
& =1+4 Q^{80}+4 Q^{100}+4 Q^{200}+16 Q^{280}+4 Q^{320}+16 Q^{360}+12 Q^{400}+\text {. } \\
& +16 Q^{480}+16 Q^{520}+16 Q^{560}+32 Q^{600}+4 Q^{640}+20 Q^{720}+44 Q^{800}+ \\
& +16 Q^{840}+16 Q^{880}+16 Q^{920}+16 Q^{960}+40 Q^{1000}+24 Q^{1040}+32\left(Q^{1080}+\right. \\
& +32 Q^{1120}+32 Q^{1160}+64 Q^{1200}+32 Q^{1240}+4 Q^{1280}+32 Q^{1320}+ \\
& +8 Q^{1360}+64 Q^{1400}+52 Q^{1440}+16 Q^{1480}+16 Q^{1520}+32 Q^{1560}+44 Q^{1600}+ \\
& +48 Q^{1640}+32 Q^{1680}+32 Q^{1720}+48 Q^{1760}+100 Q^{1800}+48 Q^{1840} \\
& +16 Q^{1880}+16 Q^{1920}+16 Q^{1960}+84 Q^{2000}+64 Q^{2040}+56 Q^{2080}+ \\
& +16 Q^{2120}+64 Q^{2160}+80 Q^{2200}+32 Q^{2240}+64 Q^{2280}+56 Q^{2320}+. \\
& +64 Q^{2360}+176 Q^{2400}+48 Q^{2440}+32 Q^{2480}+48 Q^{2520}+4 Q^{2500} \text {. } \\
& +104 Q^{2800}+64 Q^{2040}+32 Q^{2080}+72 Q^{2720}+48 Q^{2700}+112 Q^{2 \mathrm{HOO}}+ \\
& +32 Q^{2840}+52 Q^{2880}+64 Q^{2920}+56\left(Q^{2000}+16\left(0 Q^{3000}+80 Q^{3040}\right.\right. \\
& +96 Q^{3080}+64 Q^{3120}+64 Q^{3100}+44 Q^{3200}+48 Q^{9240}+72\left(Q^{3280}+\right. \\
& +96 Q^{3320}+128 Q^{3800}+136 Q^{3400}+32 Q^{3440}+64 Q^{3480}+48 Q^{3520}+ \\
& +64 Q^{3560}+188 Q^{3600}+64 Q^{3640}+96 Q^{3080}+96 Q^{3720}+48 Q^{3700} \text { ․ } \\
& +144 Q^{3800}+16 Q^{3840}+\ldots
\end{aligned}
$$

Die Entwicklungen der Funktionen $\vartheta_{00}^{2}(\tau ; 0,4) \vartheta_{40}(\tau ; 0,20) \vartheta_{12,0}(\tau ; 0,20)$, $\vartheta_{41}^{2}(\tau ; 0,20) \vartheta_{12,1}^{2}(\tau ; 0,20), \vartheta_{20,0}^{2}(\tau ; 0,20) \vartheta_{80}(\tau ; 0,20) \vartheta_{16,0}(\tau ; 0,20)$ und $\Theta(\tau ; 10)$ nach Potenzen von $Q$ sind in den Formeln (12.11), (12.13), (12.15) und (12.16) gegeben.

Man wähle jetzt die Konstanten $A_{1}, A_{2}$ und $A_{3}$ so, daß die Koeffizienten von $Q^{40}, Q^{80}$ und $Q^{280}$ in den Entwicklungen der Funktion $\psi(\tau ; 2,5)$ verschwinden. Nach (13.1), (13.3), (12.11), (12.13), (12.15) und (12.16) kommt dies darauf hinaus, daß

$$
-A_{1}-\frac{2}{3}=0, \quad 4-A_{2}-\frac{4}{3}=0, \quad 16-4 A_{1}-4 A_{3}-\frac{16}{3}=0,
$$

woraus

$$
A_{1}=-\frac{2}{3}, \quad A_{2}=\frac{8}{3}, \quad A_{3}=\frac{10}{3} .
$$

Bestimmt man folglich die Konstanten $A_{1}, A_{2}$ und $A_{3}$ durch (13.4), so läßt sich ohne Schwierigkeit nachprüfen, daß nach (13.1), (13.3), (12.11) (12.13), (12.15) und (12.16) alle Koeffizienten von $Q^{M}, M \leqslant 3840$ gleich Null sind.

SATZ 6a. Es sei $n=2^{\alpha} 5^{\beta} u,(u, 10)=1, M=40 n$. Dann ist

$$
r(n)=\left\{\begin{array}{lll}
\frac{1}{3}\left(5^{\beta+1}-3\right) \sigma(u)-\frac{2}{3} \nu_{2}(M)+\frac{10}{3} v_{4}(M) & \text { für } & \alpha=0, \\
\frac{2}{3}\left(5^{\beta+1}-3\right) \sigma(u)+\frac{8}{3} \nu_{3}(M) & \text { für } & \alpha=1, \\
2\left(5^{\beta+1}-3\right) \sigma(u) & \text { für } & \alpha>1,
\end{array}\right.
$$

wo $v_{2}(M), v_{3}(M)$ und $v_{4}(M)$ die Koeffizienten von $Q^{M}$ in den Entwicklungen der Funktionen $\vartheta_{00}^{2}(\tau ; 0,4) \vartheta_{40}(\tau ; 0,20) \vartheta_{12,0}(\tau ; 0,20), \vartheta_{41}^{2}(\tau ; 0,20) \vartheta_{12,1}^{2}(\tau ; 0,20)$ und $\vartheta_{20,0}^{2}(\tau ; 0,20) \vartheta_{80}(\tau ; 0,20) \vartheta_{16,0}(\tau ; 0,20)$ nach Potenzen von $Q$ bezeichnen.

Beweis. Die Behauptung folgt aus (13.2), (4.2), (4.3), (4.7), (12.6) und (12.21) -(12.23).

\section{Literaturverzeichnis}

[1] F. Klein, Vorlesungen über die Theorie der elliptischen Modulfunktionen, Ausgearbeitet und vervollständigt von R. Fricke, Leipzig 1890.

[2] H. D. Kloosterman, On the representation of numbers in the form $a x^{2}+b y^{2}+$ $+c z^{2}+d t^{2}$, Proceedings of the London Mathematical Society, ser. 2,25 (1925), S. 143 - 173.

[3] - Theorie der Eisensteinschen Reihen von mehreren Veränderlichen, Abhandlungen aus dem Mathematischen Seminar der Hamburgischen Universität 6 (1928), S. $163 \cdot 188$.

[4] - The behaviour of general theta functions under the modular group and the (4) binary modular congruence groups. I, Annals of Mathematics 47 (1946), S. $317-375$. 


\section{ACTA ARITHMETICA}

$\mathrm{V}(\mathbf{1 9 5 9 )}$

[5] J. Liouville, Sur la forme $x^{2}+y^{2}+5 z^{2}+5 t^{2}$, Journal de Mathómatiques, ser. 2,10 (1865), S. 1 -8.

[6] - Sur la forme $x^{2}+y^{2}+9 z^{2}+9 t^{2}$, Journal de Mathématiques, ser. 2, 10 (1865), S. 14-20.

[7] Г.А. Л о мад зе, О представлении иисел суммами обобиеньньв: полиеолальиық иисел. $I$, Труды Тбилисского математического института им. А. М. Размадзо 22 (1956), S. $77-102$

[8] H. Streefkerk, Over het aantal oplossingen der diophantische vergelijlcing $U=\sum_{i=1}^{s}\left(A x_{i}^{2}+B x_{i}+O\right)$, Amsterdam 1943.

[9] A. Walfisz, Gitterpunkte in mehrdimensionalen Kugeln, Monografie Matom matyczne T. 33, Warszawa 1957.

Regu par la Rédaction le 1\%. 6. 1958

\section{Remarks on number theory II \\ Some problems on the $\sigma$ function}

by

P. ERDös (Budapest)

H. J. Kanold and I (see [1] and [4]) observed that if $a$ and $b$, where $a \neq b$, are squarefree integers then $\sigma(a) / a \neq \sigma(b) / b$. The proof is very simple. Assume $\sigma(a) / a=\sigma(b) / b$; we can clearly assume $(a, b)=1$. Let $p$ be the greatest prime factor of $a b$, say $p \mid a, p \times b$. But then $a \sigma(b)=b \sigma(a)$ is clearly impossible, since the left side is a multiple of $p$ and the right side is not.

On the other hand the equation

$$
\frac{\sigma(a)}{a}=\frac{\sigma(b)}{b}
$$

clearly has infinitely many solutions, e. g. if $(n, 42)=1$,

$$
\frac{\sigma(6 n)}{6 n}=\frac{\sigma(28 n)}{28 n}=2 \frac{\sigma(n)}{n} .
$$

A solution of (1) is called primitive if

$$
\frac{\sigma(a)}{a}=\frac{\sigma(b)}{b} \text { but for every } d|a, d| b,
$$

$$
\left(d, \frac{a}{b}\right)=\left(d, \frac{b}{d}\right)=1, \quad \sigma\left(\frac{a}{d}\right) \neq \sigma\left(\frac{b}{d}\right),
$$

in other words $a$ and $b$ are called primitive solutions of (1) if no prime $p$ divides $a$ and $b$ to the same exponent. Clearly every solution $a_{1}, b_{1}$ of $(1)$ can be written in the form $a_{1}=a u_{1}, b_{1}=b u$ where $a$ and $b$ are primitive solutions and $(u, a b)=1$.

It is very probable that if $\left\{a_{1}, b_{1}\right\},\left\{a_{2}, b_{2}\right\}$ are primitive solutions then $a_{2}=k a_{1}, b_{2}=k b_{1}$ is impossible. 